\title{
Universal first-passage statistics in aging media
}

\author{
N. Levernier, ${ }^{1}$ O. Bénichou, ${ }^{1}$ T. Guérin, ${ }^{2}$ and R. Voituriez ${ }^{1,3}$ \\ ${ }^{1}$ Laboratoire de Physique Théorique de la Matière Condensée, CNRS, UPMC, 4 Place Jussieu, 75005 Paris, France \\ ${ }^{2}$ Laboratoire Ondes et Matière d'Aquitaine, University of Bordeaux, Unité Mixte de Recherche No. 5798, CNRS, 33400 Talence, France \\ ${ }^{3}$ Laboratoire Jean Perrin, CNRS, UPMC, 4 Place Jussieu, 75005 Paris, France
}

(Received 13 December 2017; revised manuscript received 25 May 2018; published 23 August 2018)

\begin{abstract}
It has been known for a long time that the kinetics of diffusion-limited reactions can be quantified by the time needed for a diffusing molecule to reach a target: the first-passage time (FPT). So far the general determination of the mean first-passage time to a target in confinement has left aside aging media, such as glassy materials, cellular media, or cold atoms in optical lattices. Here we consider general non-Markovian scale-invariant diffusion processes, which model a broad class of transport processes of molecules in aging media, and demonstrate that all the moments of the FPT obey universal scalings with the confining volume with nontrivial exponents. Our analysis shows that a nonlinear scaling with the volume of the mean FPT, which quantities the mean reaction time, is the hallmark of aging and provides a general tool to quantify its impact on reaction kinetics in confinement.
\end{abstract}

DOI: 10.1103/PhysRevE.98.022125

\section{INTRODUCTION}

How long does it take a random walker to find a target site? This time, usually called a first-passage time (FPT), has been extensively studied [1-3] because of its relevance to the many processes that are controlled by first-passage events. At the microscopic scale, this is the case of diffusionlimited reactions, which are controlled by the encounter of reaction partners [4]. At the macroscopic scale, random search processes, exemplified by the search for food by animals, have been shown over the past decade to be efficiently quantified by FPTs [5-9]. A first step in the analysis of FPTs consists in determining its mean, the mean first-passage time (MFPT).

Geometrical confinement is a key parameter in the evaluation of MFPTs of diffusing particles, as illustrated by the simple model of a one-dimensional (1D) or 2D symmetric random walk with nearest-neighbor jumps on a regular lattice. In the absence of confinement it is well known that a 1D or $2 \mathrm{D}$ random walker eventually visits any site of the lattice with certainty. However, due to the long-tail statistics of the FPT distribution, the MFPT turns out to be infinite in this case. In higher dimensions, the MFPT to a given site is still infinite, since the weight of trajectories that never reach it is finite. The analysis of FPT statistics for general random walks in such infinite geometries has been the focus of intense activity [10-15]. In the opposite case where the random walker is confined in a bounded domain, the situation is radically different. A target site is found with probability one and the long tails of the FPT distribution are generally suppressed, leading to a finite MFPT. The question that naturally arises is then to determine the scaling of the MFPT with the volume of the confining domain.

This cannot be determined solely by dimensional analysis since $a$ priori at least three length scales are involved [16]: the target size $a$, the source to target distance $r$, and the typical confining domain size $R \propto V^{1 / d_{f}}$, where $V$ is the volume of the confining domain and $d_{f}$ the fractal dimension of the system. However, in the case of scale-invariant Markovian processes, a general scaling of the MFPT with $a, r$, and $V$ has been derived in the large-volume limit $V \rightarrow \infty$, yielding in particular a general linear dependence on $V$ [8]. This result was further extended to higher-order moments and the full FPT distribution was determined asymptotically, leading to the concept of geometry-controlled kinetics [17].

Recently, the case of non-Markovian Gaussian processes with stationary increments was analyzed $[9,18]$ and a linear scaling with $V$ was again found for the MFPT. However, these analyses leave aside the class of general aging processes, which model diffusion of particles in aging media. Note that the particular case of continuous-time random walks (CTRWs) was shown to display aging [19] and the FPT statistics of CTRWs in confinement were derived earlier with standard methods [20-22]. As discussed below, this type of aging has no effect on the FPT statistics in the limit of large confining volume considered here. Aging media are generally characterized by the fact that physical observables depend on the time elapsed since the preparation of the system. Examples of aging processes range from the slowing down observed in glassy systems [23] to the broadening in the velocity distribution of cold atoms in optical lattices [24] or the nonstationary behavior of the mean-square displacement of tracers in the plasma membrane [25]. More generally, any random walker interacting with other degrees of freedom is expected to display memory effects, in which case aging properties, reflecting the decay of the memory of the initial state, naturally arise. Such transient aging gives rise to nontrivial persistence exponents characterizing the long-time decay of the first-passage-time distribution in infinite space [15]. In turn, the FPT problem for the target search in a confined aging medium, and therefore the understanding of reaction kinetics in these media, is not well characterized theoretically. A striking example is given by biochemical reactions in cellular media, such as gene regulation. These are known to involve a small copy number of reactants, confined in the cell or its nucleus, which diffuse in a highly dynamic medium. The resulting dynamics is therefore 
generically nonstationary (see, e.g., [25] or [26]). Another example is given by chemical reactions in supercooled liquids after a temperature quench, where aging in single-particle dynamics is routinely observed [23].

In this article, we quantify reaction kinetics in aging media by addressing the first-passage problem in confinement for a wide class of aging stochastic processes. In our analysis, the system can age at the same time the target search occurs. We show that the full FPT distribution for scale-invariant processes in confinement falls into universality classes that we define below and thereby identify the impact of aging on reaction kinetics. In addition, our approach is applied to emblematic non-Markovian processes with stationary increments such as $d$-dimensional fractional Brownian motion or Lévy walks, whose FPT properties have so far remained out of reach with standard techniques.

\section{RESULTS}

\section{A. Model}

We consider a general scale-invariant non-Markovian stochastic process defined in a space of dimension $d$ (while all examples below are defined in Euclidean spaces, our results can be generalized to fractal spaces of dimension $d_{f}$, by direct substitution $d \rightarrow d_{f}$ ). The process is assumed to be symmetric (there is no privileged direction) and its increments satisfy at long times

$$
\left\langle[X(t+\tau)-X(t)]^{2}\right\rangle \underset{t \rightarrow \infty}{\sim} t^{\alpha} \tau^{\beta},
$$

where we have introduced an aging exponent $\alpha$ which describes, for $\alpha \neq 0$, the nonstationary dynamics of increments. Here $f(t) \underset{t \rightarrow \infty}{\sim} g(t)$ stands for $f(t) / g(t) \underset{t \rightarrow \infty}{\rightarrow} A$, where $A$ is a nonzero constant. This assumption means that the measured increments during a time lag $\tau$ will be subdiffusive $(\beta<1)$ or superdiffusive $(\beta>1)$, with a generalized transport coefficient $\sim t^{\alpha}$ that depends on the time $t$ elapsed since the initial state (see Fig. 1). Hence, $\alpha>0$ corresponds qualitatively to accelerating processes and $\alpha<0$ to slowing down processes, both

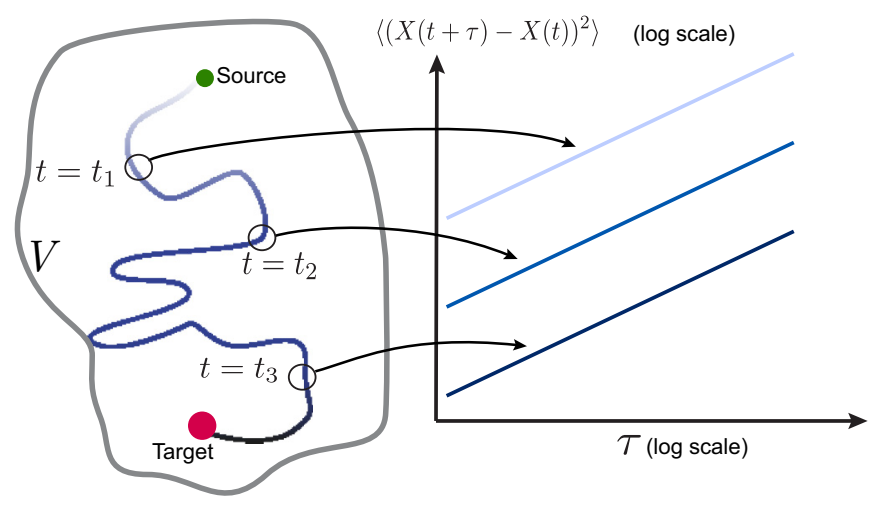

FIG. 1. First-passage times of a diffusing particle in an aging medium. A diffusing particle starting from $S$ is searching for a target $T$ in an aging medium confined in a domain of volume $V$, with reflecting boundaries. Aging is characterized by a mean-square displacement that depends on the observation time $t$. In this article, we show that reaction kinetics in aging media obeys universal statistics. cases belonging clearly to the class of aging processes. Note, however, that the above scaling (1) of increments describes only the asymptotic dynamics $(t \rightarrow \infty)$; in particular $\alpha=0$ implies that increments are asymptotically stationary, but can correspond to processes that age transiently. As we proceed to show quantitatively, even transient aging (with $\alpha=0$ ) can have important consequences on FPT statistics.

In addition, as classically done, we assume that the process itself is scale invariant and characterized by a walk dimension $d_{w}$ defined through ${ }^{1}$

$$
\left\langle X^{2 n}(t)\right\rangle \underset{t \rightarrow \infty}{\sim} t^{2 n / d_{w}} .
$$

For dimensional reasons, $d_{w}$ is found to be related to $\beta$ through $\beta=2 / d_{w}-\alpha$. Note that this definition of the walk dimension depends on the dynamics of the process and not only on the geometry of trajectories.

The scale-invariance hypotheses introduced above are consistent with the observed scaling of the dynamic structure factor discussed in the glassy literature using the examples of jammed soft materials, including colloidal gels, concentrated emulsions, and concentrated surfactant phases [27-29]. Beyond these examples, a broad range of processes, as detailed below, satisfies these hypotheses.

As was the case in the context of geometry-controlled kinetics [17], it is useful to distinguish between compact and noncompact processes that we define here by the behavior of the probability $P(a, r)=P\left(\frac{a}{r}\right)$ that in unbounded space a target of size $a$ is eventually reached by the process that started from a distance $r$. (i) If $P\left(\frac{a}{r}\right)=1$ for all $a$, the process is called compact and even a pointlike target is found with certainty $[P(0)=1]$. One then introduces the survival probability $S$ that a pointlike target has not been reached up to time $t$, which decays as $S(t) \underset{t \rightarrow \infty}{\sim} t^{-\theta}$, where the persistence exponent $\theta$ has been extensively studied in the literature [10-13,15]. Importantly, $\theta$ has been shown to depend on transient aging. Namely, using the example of fluctuating interfaces described by Edwards-Wilkinson dynamics, $\theta$ was demonstrated to take different values for different initial conditions, even if increments were asymptotically stationary $(\alpha=0$ as defined above) independently of initial conditions [12]. (ii) Conversely, if $P(0)=0$, the process is termed noncompact and will be characterized by the transience exponent $\psi$ that we introduce here and define by the small $a / r$ scaling of $P: P\left(\frac{a}{r}\right) \underset{a \rightarrow 0}{\sim}\left(\frac{a}{r}\right)^{\psi}$. (iii) Finally, the case of marginal exploration is defined here by $S(t) \sim 1 / \ln t$ for a target of radius $a \neq 0$.

\section{B. Universal first-passage statistics}

We now consider the distribution of the FPT to a target of size $a$ in a confined domain of volume $V \sim R^{d_{f}}$ (see Fig. 1), for a particle starting at a distance $r$ from the target. The origin of time corresponds to the time at which the tracer is inserted in the medium. If the observation starts at a later time $t_{1}$, it is expected that FPT statistics depend on $t_{1}$ for generic aging

\footnotetext{
${ }^{1}$ When $\left\langle X^{2 n}(t)\right\rangle$ is infinite, as in the examples of Lévy walks and Lévy flights, $d_{w}$ can be defined through the scaling of the exit time from a sphere.
} 
processes (as was shown by the example of continuous-time random walks $[20,30])$. However, in the large-volume limit that we consider below, we find that the scaling of all moments of the FPT with geometrical parameters is independent of $t_{1}$. We report that the distribution of the FPT obeys universal statistics defined as follows. Denoting by $T$ the FPT, we introduce the rescaled random variable $\eta \equiv T / T_{\text {typ }}$, where the characteristic time $T_{\text {typ }}$ is defined by

$$
T_{\text {typ }}= \begin{cases}R^{d_{w}} & \text { (compact) } \\ R^{d_{w}}\left(\ln \frac{R}{a}\right)^{1-\alpha d_{w} / 2} & \text { (marginally compact) } \\ R^{d_{w}}\left(\frac{R}{a}\right)^{\psi\left(1-\alpha d_{w} / 2\right)} & \text { (noncompact). }\end{cases}
$$

Assuming that the mean FPT to the target is finite (processes leading to infinite MFPTs, such as CTRWs with infinite mean waiting times, are analyzed in Appendix A 3), we find that $\eta$ is asymptotically distributed in the large-volume limit according to

$$
G(\eta ; a, r, R)= \begin{cases}h(\eta)\left(\frac{r}{R}\right)^{d_{w} \theta} & \text { (compact) } \\ h(\eta) \frac{\ln \frac{r}{a}}{\ln \frac{R}{a}} & \text { (marginally compact) } \\ h(\eta)\left[1-C\left(\frac{a}{r}\right)^{\psi}\right] & \text { (noncompact), }\end{cases}
$$

where it is assumed that $a \ll r, h(\eta)$ is an undetermined scaling function a priori process dependent, and $C$ is a numerical constant. We stress that both $C$ and $h$ do not depend on geometric parameters $a, r$, and $R$. Here the large-volume limit is defined by taking all points of the boundary to infinity with fixed starting position and target. In this limit FPT statistics therefore depend on the volume of the confining domain, but not its shape. In addition, it is argued in Appendix A 6 that

$$
\psi=d_{f}-\frac{d_{w}}{1-\alpha d_{w} / 2} .
$$

This explicitly determines the dependence on the geometrical parameters of the problem of the FPT distribution and therefore of all its moments (when they exist). In particular, the scaling of the mean is given by

$$
\langle T\rangle \sim \begin{cases}R^{d_{w}(1-\theta)} r^{d_{w} \theta} & \text { (compact) } \\ \frac{R^{d_{w}}}{(\ln R / a)^{\alpha d_{w} / 2}} \ln \frac{r}{a} & \text { (marginally compact) } \\ \frac{R^{d_{w}+\psi\left(1-\alpha d_{w} / 2\right)}}{a^{\psi\left(1-\alpha d_{w} / 2\right)}}\left[1-C\left(\frac{a}{r}\right)^{\psi}\right] & \text { (noncompact). }\end{cases}
$$

Remarkably, the scaling of the MFPT can therefore be nonlinear with the volume $V \sim R^{d_{f}}$ for nontrivial values of the persistence exponent $\theta$ (in the compact case) or of the aging exponent $\alpha$ (for noncompact processes). This will be the case of generic processes with nonstationary increments, as discussed below.

\section{Sketch of the proof}

We now sketch the demonstration of this result (see Appendix A for details) and first consider the compact case, for which $a$ can be taken equal to zero as stated above. We write the FPT distribution $F(t)$ as a partition over trajectories reaching either the reflecting boundary before the target [with probability $\pi(r, R)$ and conditional FPT distribution $F_{b}$ ] or the target first [with probability $1-\pi(r, R)$ and conditional FPT distribution $F_{t}$ ]: $F=\pi F_{b}+(1-\pi) F_{t}$. We now evaluate each of the terms of this equation. We remark that $1-\pi$ can be written as the time integral of the FPT density to the target restricted to trajectories reaching the target before the boundary. Most of these events occur within the typical timescale $R^{d_{w}}$ needed to reach the boundary, so we claim that the equation

$$
1-\pi(r, R) \underset{R \gg r}{\sim} \int_{0}^{A R^{d_{w}}} F_{\infty}(t, r) d t
$$

holds, where $F_{\infty}(t, r)$ is the FPT density in unconfined space and $A$ is a constant.

Using the above-mentioned hypothesis of scale invariance of the process (2), which leads in the large- $t$ limit to $F_{\infty}(t, r) \equiv$ $f\left(t / r^{d_{w}}\right) / t$ (where $f$ is an unknown process-dependent function), together with the definition of $\theta$, we obtain

$$
\pi(r, R) \underset{R \gg r}{\sim}\left(\frac{r}{R}\right)^{d_{w} \theta} .
$$

Note that here aging properties are encompassed by the persistence exponent $\theta$ [12]. While the argument leading to Eqs. (7) and (8) is not mathematically rigorous, we claim that Eq. (8) is exact. This is verified numerically in all tested examples (see Fig. 3); in addition, this equation extends the one-dimensional result for splitting probabilities of Majumdar et al. [31]. The above argument also implies that

$F_{t}(t, r, R) \sim Y\left(A R^{d_{w}}-t\right) F_{\infty}(t, r) \sim Y\left(A-\frac{t}{R^{d_{w}}}\right) \frac{r^{d_{w} \theta}}{t^{\theta+1}}$,

where $Y$ stands for the Heaviside step function. Making use again of scale invariance and writing for $R \gg r$ the exact scaling relation

$$
F_{b}(t, R) \equiv g\left(t / R^{d_{w}}\right) / t,
$$

where $g$ is an unknown function, we finally obtain Eq. (4) above.

We now turn to the noncompact case. We define by excursion a portion of trajectory starting from the sphere $S$ of radius $R / 2$ centered on the target, reaching the boundary and returning to $S$. We write the FPT distribution as a partition over the number $n$ of excursions before the first passage to the target and denote by $\Phi_{n}(t)$ the corresponding conditional FPT distribution

$$
F(t, a, r, R)=p_{0} \Phi_{0}(t)+\sum_{n=1}^{\infty} \Phi_{n}(t)\left(1-p_{0}\right)(1-p)^{n-1} p
$$

where $p \sim(a / R)^{\psi}$ is the probability to reach the target before the boundary starting from $S$ and $p_{0} \sim(a / r)^{\psi}$ is the probability to reach the target before the boundary starting from $r$. Note that here we implicitly assume that excursions are independent in the large- $R$ limit. Physically, it originates from the divergence with $R$ of the typical time $\tau_{n}$ needed to perform the $n$th excursion, which hence can be taken larger than all correlation times of the process, as was checked numerically 

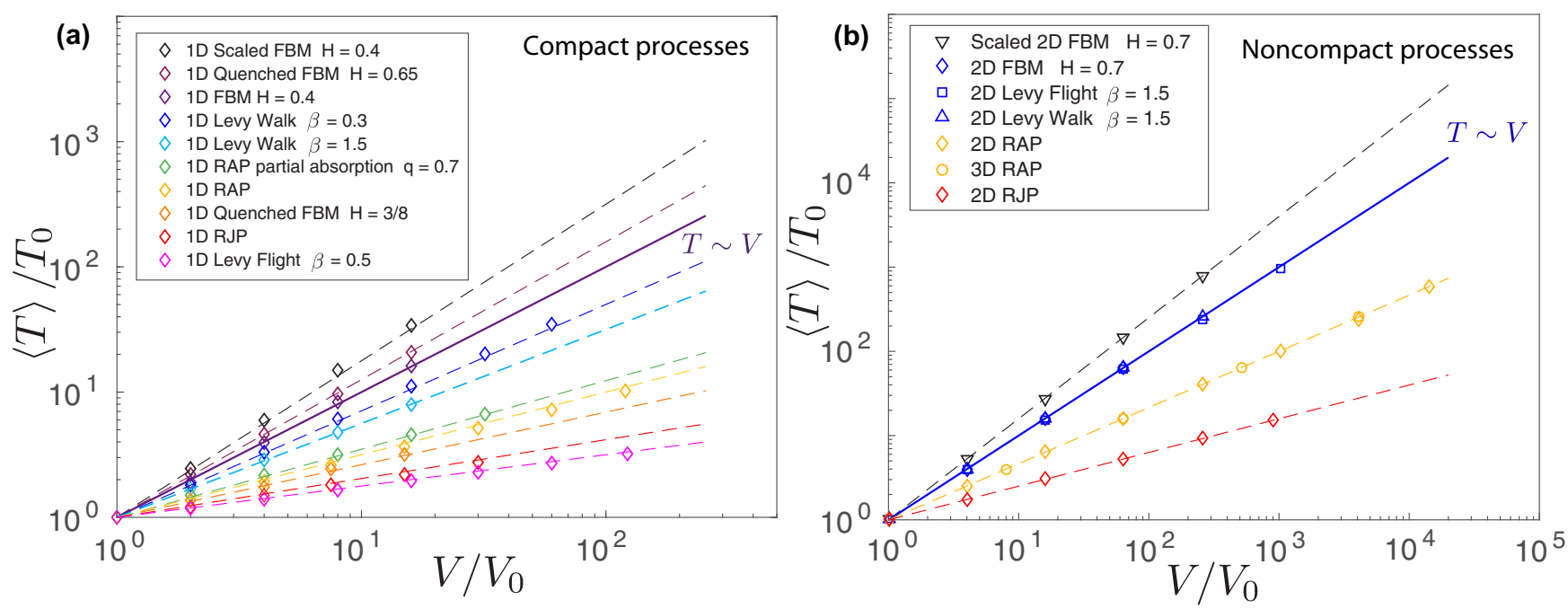

FIG. 2. Mean FPT as a function of the volume for (a) compact and (b) noncompact processes. Lines represent our predictions [Eq. (6)] and symbols are the results of numerical simulations. For each process, $\langle T\rangle$ and $V$ are rescaled by arbitrary factors $\left(V_{0}, T_{0}\right)$; $V_{0}$ is chosen big enough to have reached the large-volume limit. The solid line corresponds to the linear behavior with the volume. Details on simulation algorithms and geometrical parameters for each process are given in Appendix B.

(see Appendix A 2). Finally, a scaling argument confirmed by numerical simulations (see Appendix A 2) shows that

$$
\Phi_{n}(t)=\frac{1}{t} \phi\left(t / t_{n}\right),
$$

where $t_{n}$ is the typical time elapsed after $n$ excursions, which satisfies

$$
\left\langle\left[X\left(t_{n}+\tau_{n}\right)-X\left(t_{n}\right)\right]^{2}\right\rangle \underset{t_{n} \rightarrow \infty}{\sim} t_{n}^{\alpha} \tau_{n}^{2 / d_{w}-\alpha}=R^{2} .
$$

Using that $t_{n}=\tau_{1}+\cdots+\tau_{n}$, this equation leads to

$$
t_{n} \underset{R \rightarrow \infty}{\sim} R^{d_{w}} n^{1-\alpha d_{w} / 2} .
$$

Making use of the definition of $\psi$, the large- $R$ behavior of (11) is found to lead to Eq. (4) above. Note that, as soon as $\alpha \neq 0$, the typical time $t_{n}$ explicitly depends on $n$, which is a direct manifestation of aging.

\section{Remark: The case of CTRWs}

For completeness, we describe in this section how the FPT statistics of CTRWs (which have been derived earlier with standard tools [20-22]) can be obtained in the general framework introduced above (for details, see Appendix A 3). Continuous-time random walks are jump processes characterized by random waiting times between successive steps. For power-law distributions of waiting times $q(t) \sim 1 / t^{\beta+1}$ with $\beta<1$, the mean waiting time is infinite. The process is in this case subdiffusive $\left(d_{w}=2 / \beta\right)$ and increments satisfy $\left\langle[X(t+\tau)-X(t)]^{2}\right\rangle \underset{t \rightarrow \infty}{\sim} t^{\beta-1} \tau[19] ;$ in that sense aging therefore occurs.

In the compact case, the formalism introduced above applies directly. For example, for $d=1$, using $\theta=\beta / 2$ [15] in Eqs. (3) and (4), one recovers the results of [20-22]. In the noncompact case, there is a subtlety in the definition of the aging exponent $\alpha$. The quantity needed to derive FPT properties is the exponent that characterizes aging in the duration of excursions (discussed in the preceding section), which begin just after a jump. The variables $t$ and $t+\tau$ in the definition of increments are therefore conditioned by the fact that a jump just occurred at time $t$. With this prescription, we find that the conditioned increments satisfy $\left\langle[X(t+\tau)-X(t)]^{2}\right\rangle_{c} \sim \tau^{\beta}$, so one must set $\alpha=0$ for a CTRW. Note that this contrasts with the unconditioned increments given above. Making use of this definition of $\alpha=0$, together with Eqs. (3) and (4), the results of [20-22] are recovered. Finally, this shows that FPT statistics of CTRWs can be recovered by our approach.

\section{DISCUSSION}

\section{A. Dependence of reaction kinetics on the geometrical parameters}

We now comment on the main results of this paper, Eqs. (3)-(6). First, the dependence on $a, r$, and $R$ of the FPT distribution of Markovian processes [17] and the mean FPT of non-Markovian Gaussian processes with stationary increments [9] are recovered. This results from the specific values of $\alpha$ and $\theta$ for processes with stationary increments: $\alpha=0$ by definition of stationary increments, which leads to $\psi=d_{f}-d_{w}$ for noncompact processes; for compact processes, we argue in Appendix A 5 that $\theta=1-d_{f} / d_{w}$.

Second, a remarkable feature emerging with aging is a possible nonlinear scaling of the MFPT, and thus of the mean reaction time, with the volume either sublinear or superlinear (see Fig. 2). A linear scaling is shown to hold in the compact case only if increments are stationary at all times (leading to $\theta=1-d_{f} / d_{w}$ ), while in the noncompact case asymptotically stationary increments (leading to $\alpha=0$ ) are sufficient. For example, a non-Markovian compact process, which is initially quenched and relaxes to its stationary state in free space, therefore displaying aging only transiently $\left(\theta \neq 1-d_{f} / d_{w}\right.$ and $\alpha=0$ ), is shown below to be characterized by a nonlinear scaling of the MFPT with the volume (see Fig. 2). By contrast, in noncompact cases, a nonlinear scaling can be obtained only if aging occurs at all times. 

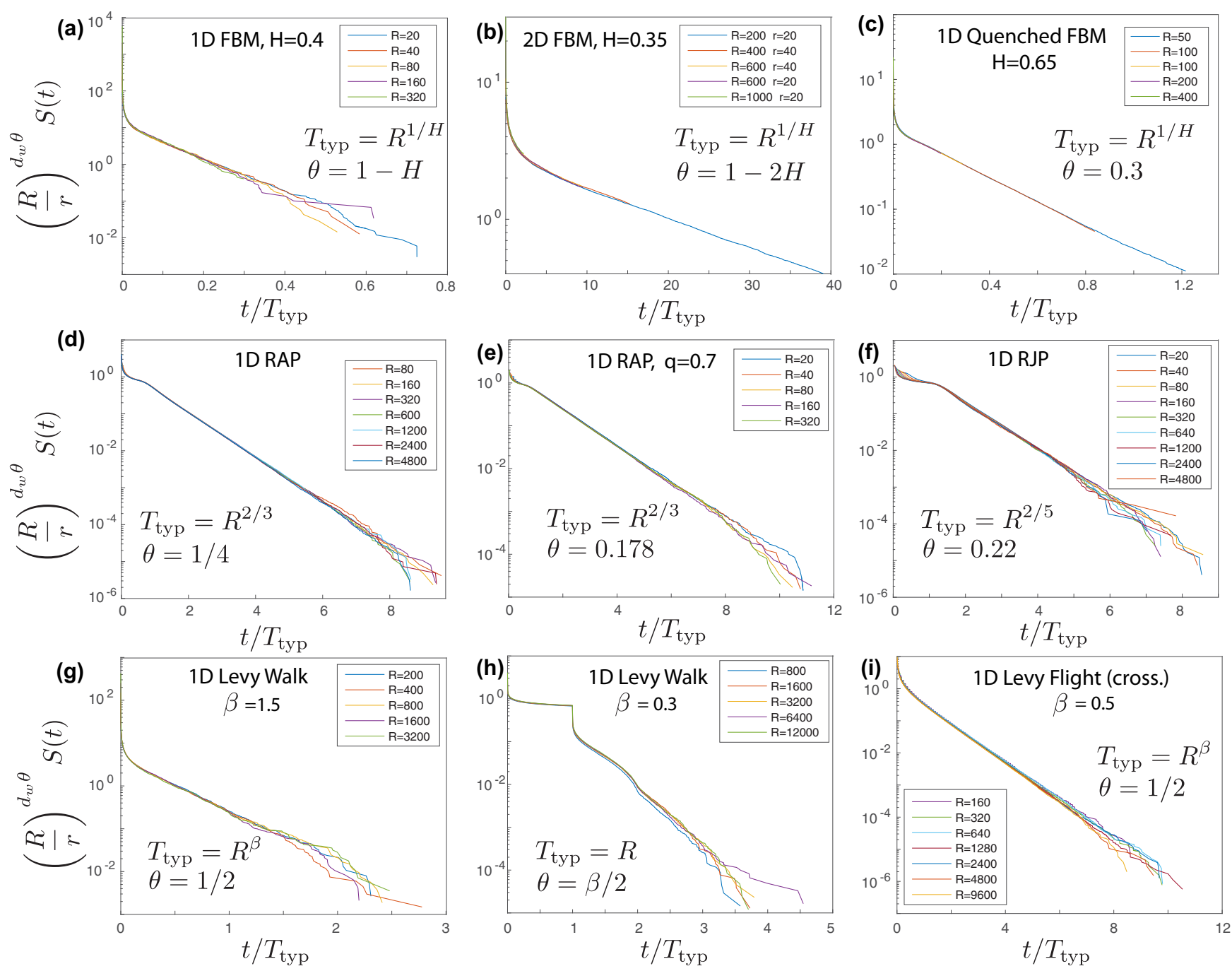

FIG. 3. Universal scalings of the FPT density with geometrical parameters for compact processes. Here $S \equiv \int_{t / T_{\mathrm{typ}}}^{+\infty} G(\eta) d \eta$ is the survival probability of the random walker, whose scaling with geometrical parameters is deduced from Eqs. (3) and (4). The collapse of numerical data after rescaling for different geometrical parameters shows that our theory unambiguously captures the dependence of the FPT distribution on geometrical parameters. Simulations are performed in 1D or 2D boxes of size $R$ with periodic boundary conditions: (a) 1D fractional Brownian motion with $H=0.4$, (b) $2 \mathrm{D}$ fractional Brownian motion with $H=0.35$, (c) $1 \mathrm{D}$ initially quenched fractional Brownian motion with $H=0.65$, (d) $1 \mathrm{D}$ random acceleration process, (e) $1 \mathrm{D}$ random acceleration process with a probability $q=0.7$ of absorption at each crossing of the target, (f) 1D random jerk process, (g) 1D Lévy walk ( $X Y$ convention) whose jumps are Lévy stable distributed with a parameter $\beta=1.5$, (h) $1 \mathrm{D}$ Lévy walk ( $X Y$ convention) whose jumps are Lévy stable distributed with a parameter $\beta=0.3$, and (i) 1D Lévy flight ( $X Y$ convention) whose jumps are Lévy stable distributed with a parameter $\beta=0.3$ and the target is found when crossed. Details on the definition of the processes, the simulation algorithms, and the geometrical parameters for each process are given in Appendix B.

Third, the FPT statistics displays a strong dependence on the initial distance in the case of compact exploration and a much weaker one in the noncompact case. Qualitatively, this feature is the same as that previously found for Markovian processes $[8,17]$, but aging quantitatively modifies the exponents characterizing this dependence.

Next, note that the timescale $T_{\text {typ }}$ of the FPT distribution is independent of $\theta$ and $\alpha$ that characterize transient and asymptotic aging for compact processes, while it depends explicitly on $\alpha$ for noncompact processes.

Finally, the dependence of the FPT distribution on $r, a$, and $R$ falls into universality classes defined by $d_{w}$ and $\theta$ (for compact processes) or by $\alpha, d_{w}$, and $d_{f}$ (for noncompact processes), exponents being an input of the model. Note that, in the compact case, the dependence on aging is entirely contained in the exponent $\theta$, with no explicit dependence on $\alpha$.

\section{B. Examples and numerical simulations}

We finally confirm the validity of our analytical results by comparing them to numerical simulations of a broad range of representative examples of stochastic processes (see Appendix $\mathrm{B}$ for details), for which only sparse results on the FPT statistics in confinement were available so far [9,20,30,32-34]. These processes all satisfy the scale-invariance hypothesis introduced above, with the exception of Lévy walks for which multifractal scalings can occur, as discussed in Appendix B 6. Specifically, 

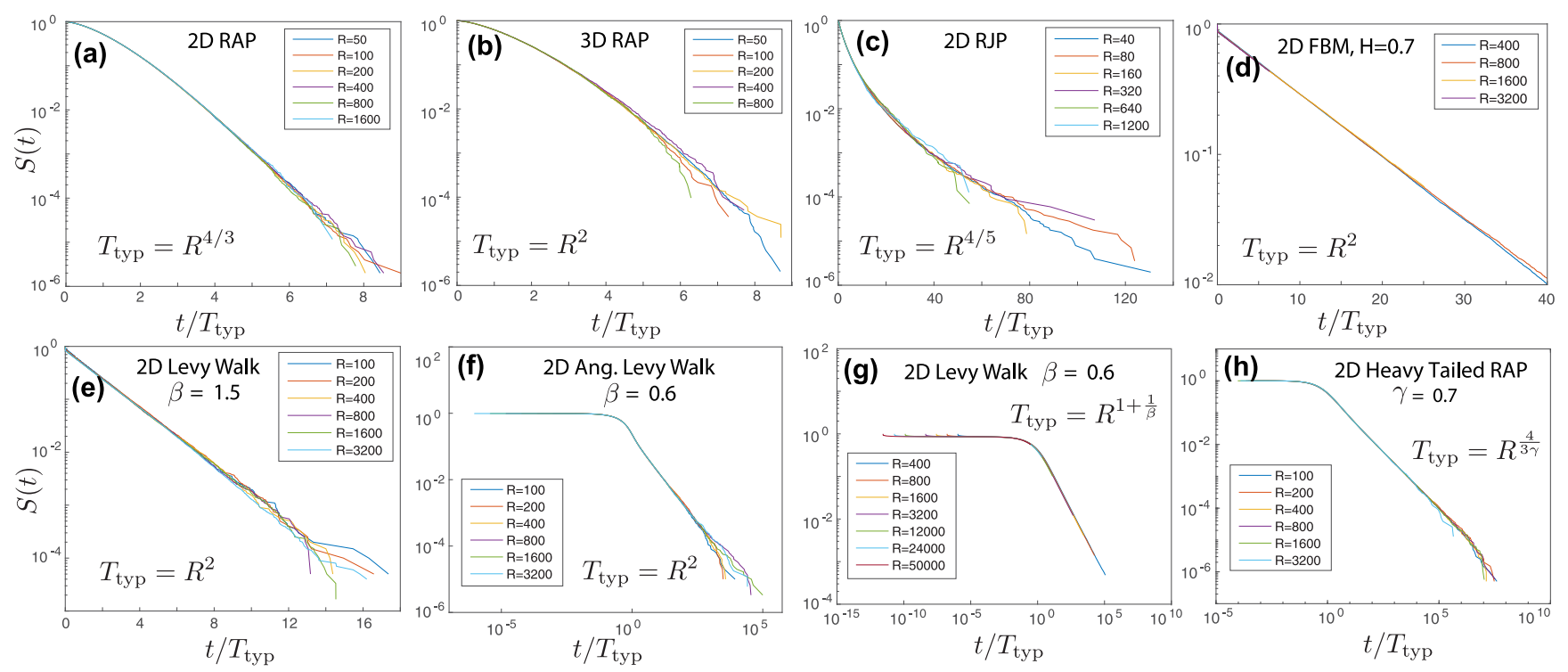

FIG. 4. Universal scalings of the FPT density with geometrical parameters for noncompact processes. Here $S \equiv \int_{t / T_{\text {typ }}}^{+\infty} G(\eta) d \eta$ is the survival probability of the random walker, whose scaling on geometrical parameters is deduced from Eqs. (3) and (4). The collapse of numerical data after rescaling for different geometrical parameters shows that our theory unambiguously captures the dependence of the FPT distribution on geometrical parameters. Simulations are performed in 1D or 2D boxes of size $R$ with periodic boundary conditions, except for the stubborn RAP (see Appendix B for this particular case): (a) 2D random acceleration process, (b) 3D random acceleration process, (c) 2D random jerk process, (d) 2D fractional Brownian motion with $H=0.7$, (e) 2D Lévy walk ( $X Y$ convention) whose jumps are Lévy stable distributed with a parameter $\beta=1.5$, (f) 2D Lévy walk (angular convention) whose jumps are Lévy stable distributed with a parameter $\beta=0.6$, (g) 2D Lévy walk ( $X Y$ convention) whose jumps are Lévy stable distributed with a parameter $\beta=0.6$, and (h) so-called 2D heavy-tailed RAP, with a CTRW parameter $\gamma=0.7$. Graphs (g) and (h) cover the case of infinite mean FPT, for which Eqs. (1) and (3) have to be corrected, as explained in Appendix A 3. Details on the processes, the simulation algorithms, and the geometrical parameters for each process are found in Appendix B.

we consider (i) the $d$-dimensional fractional Brownian motion (FBM), a non-Markovian Gaussian process of constant mean, with stationary increments satisfying $\left\langle[X(t)-X(0)]^{2}\right\rangle=t^{2 H}$, where $H$ is the Hurst exponent; this process has been repeatedly invoked in the literature to model anomalous diffusion arising from the interaction with many variables [12,14]; (ii) its extension to quenched initial conditions, for which increments are time dependent and relax asymptotically to the stationary behavior; for this process, the aging exponent vanishes, but the progressive decay of the memory of the initial state makes the increments nonstationary at intermediate timescales and the persistence exponent nontrivial [12] (in the compact case); (iii) the $d$-dimensional random acceleration process (RAP), defined by $\ddot{X}=\eta(t)$ with $\eta(t)$ a Gaussian white noise, often considered as the simplest, yet nontrivial, non-Markovian process [15,34,35]; (iv) the generalizations of the RAP to the case of partial absorption, various conditions at the confining boundary, higher-order derivatives such as the random jerk process (RJP) [satisfying $\dddot{X}=\eta(t)$ ], and potential long waiting times; (v) $d$-dimensional Lévy flights, where at each time step the random walker performs a jump whose size $l$ is drawn from a long-tailed distribution $p(l) \sim 1 / l^{1+\beta}$, with both prescriptions of first arrival and first crossing of the target [36]; (vi) $d$-dimensional Lévy walks, which can be described as Lévy flights with a finite velocity; scale-invariance properties of this process are discussed in Appendix B 6; and (vii) scaled processes defined from a reference process $X^{(0)}(t)$ by $X(t) \equiv X^{(0)}\left(t^{b}\right)$. These cases cover a broad class of stochastic processes in one and higher dimensions, which can be aging [processes (ii)-(iv) and (vii)] or not [processes (i), (v), and (vi)], Markovian or non-Markovian, subdiffusive or superdiffusive. Note that even for nonaging processes, most of the available results concerning non-Markovian processes were so far limited to $d=1$.

Figures 2-4 reveal excellent quantitative agreement between numerical simulations and our analytical results. The data collapse of the properly rescaled FPT distribution shows that our approach unambiguously captures the dependence on $a$ and $r$ and on $R$ for both compact (Fig. 3) and noncompact (Fig. 4) processes. In particular, sublinear, linear, and superlinear scalings of the mean FPT with the volume are observed, in agreement with our predictions (Fig. 2). This demonstrates that the nonlinear scaling of the mean FPT with the volume is the hallmark of the aging properties of the dynamics. A summary of our predictions for all these processes can be found in Tables I and II.

\section{CONCLUSION}

Our results provide a framework to quantify the kinetics of reactions in aging environments. Using the example of biochemical reactions in cellular media or chemical reactions in supercooled liquids, a typical protocol would consist in measuring the exponents $d_{w}, \alpha$, and $\theta$ defined in the text (for example, from single-particle trajectories). The evaluation of the mean, typical, and more generally all moments of the FPT is then explicitly obtained from our expressions (3)-(6). In particular, the volume dependence, and thus the order of magnitude of the FPT, is shown to be controlled by the aging properties. Finally, we have shown how aging properties 
TABLE I. Compact processes. In all these examples, compactness of the walk requires $d=1$ except for the FBM where $d$ satisfies $d<1 / H$ and the CTRW where $d<d_{w}^{0}$. The comment "cross." for Lévy flights denotes that the first crossing of the target is investigated. Cases for which $\alpha=0$ and aging is irrelevant correspond to processes (CTRW and Lévy walks) where aging does exist but the determination of FPT statistics involves the increments conditioned by the fact that a step has just been made at time $t$, for which one finds $\alpha=0$. See Appendixes A and $\mathrm{B}$ for more details.

\begin{tabular}{|c|c|c|c|c|c|c|}
\hline Process & $d_{w}$ & $\alpha$ & Aging & $\theta$ & $T_{\text {typ }}$ & $\langle\mathbf{T}\rangle$ \\
\hline FBM $H$ & $1 / H$ & 0 & no & $1-H$ & $R^{1 / H}$ & $R^{d} r^{1 / H-d}$ \\
\hline quenched FBM $H$ & $1 / H$ & 0 & transient & $\theta(H)$ & $R^{1 / H}$ & $R^{(1-\theta) / H} r^{\theta / H}$ \\
\hline RAP & $2 / 3$ & 1 & yes & $1 / 4$ & $R^{2 / 3}$ & $R^{1 / 2} r^{1 / 6}$ \\
\hline RAP with partial absorption $q$ & $2 / 3$ & 1 & yes & $\frac{1}{4}\left[1-\frac{6}{\pi} \sin ^{-1}\left(\frac{1-q}{2}\right)\right]$ & $R^{2 / 3}$ & $R^{2(1-\theta) / 3} r^{2 \theta / 3}$ \\
\hline stubborn RAP & $2 / 3$ & 1 & yes & $1 / 4$ & $R^{2 / 3}$ & $\infty$ \\
\hline RJP & $2 / 5$ & 3 & yes & 0.2202 & $R^{2 / 5}$ & $R^{2(1-\theta) / 5} r^{2 \theta / 5}$ \\
\hline Lévy flight $\beta$ (cross.) & $\beta$ & 0 & no & $1 / 2$ & $R^{\beta}$ & $R^{1-\beta / 2} r^{\beta / 2}$ \\
\hline Lévy walk $\beta<1$ & 1 & 0 & irrelevant & $\beta / 2$ & $R$ & $R^{\beta / 2} r^{1-\beta / 2}$ \\
\hline Lévy walk $\beta>1$ & $\beta$ & 0 & no & $1 / 2$ & $R^{\beta}$ & $R^{1-\beta / 2} r^{\beta / 2}$ \\
\hline CTRW $d_{w}^{(0)}$ for $\gamma<1$ & $d_{w}^{(0)} / \beta$ & 0 & irrelevant & $\beta\left(1-d / d_{w}^{(0)}\right)$ & $R^{d_{w}^{(0)} / \beta}$ & $\infty$ \\
\hline
\end{tabular}

modify reaction kinetics and provided an explicit method to determine quantitatively FPT statistics in confined aging media. In turn, we suggest that measuring the scaling of the mean FPT with the confining volume (linear or not) could help in identifying aging properties of a medium.

\section{APPENDIX A: DETAILED DERIVATION OF THE SCALING FORM OF THE FPT DISTRIBUTION (1)-(4)}

\section{Detailed derivation of the scaling form of the FPT distribution in the compact case}

We derive here in detail the scaling form taken by the FPT distribution in the compact case. As explained in the main text, in the compact case, the radius $a$ of the target can be taken equal to zero when we focus on the limit $a \ll r$. In this section, we thus have $a=0$.

Our starting point consists in writing the FPT distribution $F$ as a partition over trajectories reaching either the reflecting boundary before the target [with probability $\pi(r, R)$ and con- ditional FPT distribution $F_{b}$ ] or the target first [with probability $1-\pi(r, R)$ and conditional FPT distribution $\left.F_{t}\right]$ :

$$
F(t, r, R)=\pi F_{b}(t, r, R)+(1-\pi) F_{t}(t, r, R) .
$$

We now evaluate each of the terms of this equation.

We remark that $1-\pi$ can be written as the time integral of the FPT density to the target restricted to trajectories reaching the target before the boundary. Most of these events occur within the typical timescale $R^{d_{w}}$ needed to reach the boundary, so one can write

$$
1-\pi(r, R) \underset{R \gg r}{\sim} \int_{0}^{A R^{d_{w}}} F_{\infty}(t, r) d t,
$$

where $A$ is a constant and $F_{\infty}(t, r)$ is the FPT density in unconfined space. Using the scale invariance of the process, we can write $F_{\infty}$ under the form

$$
F_{\infty}(t, r) \equiv \frac{1}{t} f\left(\frac{t}{r^{d_{w}}}\right),
$$

TABLE II. Noncompact processes. The dimension of space $d$ always satisfies $d>1$ except for FBM where $d>1 / H$ and a CTRW where $d>d_{w}^{0}$. Cases for which $\alpha=0$ and aging is irrelevant correspond to processes (CTRW and Lévy walks) where aging does exist but the determination of FPT statistics involves the increments conditioned by the fact that a step has just been made at time $t$, for which one finds

\begin{tabular}{|c|c|c|c|c|c|c|}
\hline Process & $d_{w}$ & $\alpha$ & Aging & $\psi$ & $T_{\text {typ }}$ & $\langle\mathbf{T}\rangle$ \\
\hline FBM $H$ & $1 / H$ & 0 & no & $d-1 / H$ & $R^{d}$ & $R^{d} a^{1 / H-d}$ \\
\hline quenched FBM $H$ & $1 / H$ & 0 & transient & $d-1 / H$ & $R^{d} a^{1 / H-d}$ & $R^{d} a^{1 / H-d}$ \\
\hline RAP & $2 / 3$ & 1 & yes & $d-1$ & $R^{2 d / 3} a^{-2(d-1) / 3}$ & $R^{2 d / 3} a^{-2(d-1) / 3}$ \\
\hline RAP partial absorption $q$ & $2 / 3$ & 1 & yes & $d-1$ & $R^{2 / 3} a^{-2(d-1) / 3}$ & $R^{2 d / 3} a^{-2(d-1) / 3}$ \\
\hline heavy-tailed RAP $\gamma<1$ & $2 / 3 \gamma$ & $\gamma$ & yes & $d-1$ & $R^{2 /(3 \gamma)} a^{-2(d-1) /(3 \gamma)}$ & $\infty$ \\
\hline RJP & $2 / 5$ & 3 & yes & $d-1$ & $R^{2 / 5} a^{-2(d-1) / 5}$ & $R^{2 d / 5} a^{-2(d-1) / 5}$ \\
\hline Lévy flight $\beta$ (arr.) & $\beta$ & 0 & no & $d-\beta$ & $R^{d} a^{\beta-d}$ & $R^{d} a^{\beta-d}$ \\
\hline Lévy flight $\beta>1$ (cross.) & $\beta$ & 0 & no & $d-\beta$ & $R^{d} a^{\beta-d}$ & $R^{d} a^{\beta-d}$ \\
\hline Lévy flight $\beta<1$ (cross.) & $\beta$ & 0 & no & $d-1$ & $R^{d+\beta-1} a^{1-d}$ & $R^{d+\beta-1} a^{1-d}$ \\
\hline Lévy walk $(X Y) \beta<1$ & 1 & 0 & irrelevant & $d-1$ & $R^{1+(d-1) / \beta} a^{(1-d) / \beta}$ & $\infty$ \\
\hline Lévy walk $(X Y) \beta>1$ & $\beta$ & 0 & no & $d-\beta$ & $R^{d} a^{\beta-d}$ & $R^{d} a^{\beta-d}$ \\
\hline Lévy walk (angular) $\beta<1$ & 1 & 0 & irrelevant & $d-1$ & $R^{d} a^{1-d}$ & $R^{d} a^{d-1}$ \\
\hline CTRW $d_{w}^{(0)}$ for $\gamma<1$ & $d_{w}^{(0)} / \beta$ & 0 & irrelevant & $d-d_{w}^{(0)}$ & $R^{d / \beta} a^{d / \beta-d_{w}^{(0)} / \beta}$ & $\infty$ \\
\hline
\end{tabular}
$\alpha=0$. In the case of Lévy flights "arr." indicates that the convention of first arrival is used, whereas "cross." indicates the first crossing. Finally, both $X Y$ and angular Lévy walks are analyzed. See Appendixes A and B for more details. 
where $f$ is a dimensionless function. Using Eq. (A3) and the fact that $F_{\infty}$ is normalized to unity, we obtain, from Eq. (A2),

$$
\pi(r, R) \underset{R \gg r}{\sim} \int_{A R^{d_{w}}}^{\infty} \frac{1}{t} f\left(\frac{t}{r^{d_{w}}}\right) d t .
$$

Next, using the definition of the persistence exponent, we find that the long-time behavior of the function $f$ satisfies

$$
f\left(\frac{t}{r^{d_{w}}}\right) \underset{t \rightarrow \infty}{\sim}\left(\frac{r^{d_{w}}}{t}\right)^{\theta} .
$$

Inserting this relation into the integral (A4), we finally obtain the following scaling for the probability $\pi$ of touching the boundaries of the confining domain before the target:

$$
\pi(r, R) \underset{R \gg r}{\sim}\left(\frac{r}{R}\right)^{d_{w} \theta} .
$$

Note that the above relation extends the one-dimensional result of [31]. Similarly, we can write the FPT distribution to the target $F_{t}$, conditional on the fact that the target is hit before the domain boundaries, as

$$
\begin{aligned}
F_{t}(t, r, R) & \sim Y\left(A R^{d_{w}}-t\right) F_{\infty}(t, r) \\
& \sim Y\left(A-\frac{t}{R^{d_{w}}}\right) \frac{f\left(t / r^{d_{w}}\right)}{t},
\end{aligned}
$$

where $Y$ stands for the Heaviside step function.

Next, $F_{b}$ is the conditional FPT distribution for trajectories that hit the domain boundaries first, we can thus argue that it does not depend on $r$. Making use again of scale invariance, we obtain

$$
F_{b}(t, r, R) \equiv \frac{1}{t} g\left(\frac{t}{R^{d_{w}}}\right) .
$$

Collecting all terms, we obtain

$$
\begin{aligned}
F(t, r, R) \underset{R \gg r}{\sim} & \left(\frac{r}{R}\right)^{d_{w} \theta} \frac{1}{t} g\left(\frac{t}{R^{d_{w}}}\right) \\
& +Y\left(A-\frac{t}{R^{d_{w}}}\right) \frac{1}{t} f\left(\frac{t}{r^{d_{w}}}\right) .
\end{aligned}
$$

We consider timescales larger than $r^{d_{w}}$ and obtain

$$
\begin{aligned}
F(t, r, R) \underset{R \gg r}{\sim} & \left(\frac{r}{R}\right)^{d_{w} \theta} \frac{1}{t} g\left(\frac{t}{R^{d_{w}}}\right) \\
& +Y\left(A-\frac{t}{R^{d_{w}}}\right) \frac{1}{t}\left(\frac{r^{d_{w}}}{t}\right)^{\theta} .
\end{aligned}
$$

Using that the distribution $G$ of the rescaled variable $\eta \equiv$ $T / R^{d_{w}}$ is given by

$$
G(\eta, r, R)=F(t, r, R) R^{d_{w}},
$$

we finally obtain that $G$ given by Eq. (A10) can be written as

$$
G(\eta, r, R)=\left(\frac{r}{R}\right)^{d_{w} \theta} h(\eta),
$$

where $h(\eta)=\left[g(\eta)+Y(A-\eta) / \eta^{\theta}\right] / \eta$ is an undetermined scaling function that is a priori process dependent.

\section{Detailed derivation of the scaling form of the FPT distribution in the noncompact case}

We derive here in detail the scaling form taken by the FPT distribution in the noncompact case. Note that we assume here that the mean FPT to the target is finite (in Appendix A 3 we describe how the results are modified in the case of infinite MFPTs).

We define by excursion a portion of trajectory starting from the sphere $S$ of radius $R / 2$ centered on the target, reaching the boundary and returning to $S$. Let us denote by $\Phi_{n}(t)$ the probability density to reach the target at time $t$ given that $n$ excursions have been performed. We write the FPT distribution as a partition over the number $n$ of excursions

$$
F(t, a, r, R)=p_{0} \Phi_{0}(t)+\sum_{n=1}^{\infty} \Phi_{n}(t)\left(1-p_{0}\right)(1-p)^{n-1} p,
$$

where $p$ is the probability to reach the target before the boundary starting from $S$ and $p_{0}$ is the probability to reach the target before the boundary starting from $r$. Note that here we implicitly assume that the probability of reaching the target after $n$ excursions is independent of the previous excursions in the large- $R$ limit. Physically, it originates from the divergence with $R$ of the typical time $\tau_{n}$ needed to perform the $n$th excursion, which hence can be taken larger than all correlation times of the process. This geometrical law of the number of excursions before reaching the target was checked numerically for two highly correlated and aging processes: the random acceleration and the random jerk processes defined in Appendix B (Fig. 5).

The probability to find the target during the $n$th excursion is equal to the probability to find the target of radius $a$ before the domain boundary, starting from a distance $R / 2$ from the target. By definition of the transience exponent $\psi$ introduced in the main text, this probability scales as

$$
p \underset{R \gg a}{\sim} C\left(\frac{a}{r}\right)^{\psi} .
$$

Similarly,

$$
p_{0} \underset{r \gg a}{\sim} C\left(\frac{a}{r}\right)^{\psi} .
$$

We now determine the scalings of the typical time $t_{n}$ at which the $n$th excursion takes place, and the typical time $\tau_{n}$ between the $n$th and the $(n+1)$ th excursion. These times can be found by noting that, during $\tau_{n}$ the typical traveled distance is $R$, which means that

$$
\left\langle\left[X\left(t_{n}+\tau_{n}\right)-X\left(t_{n}\right)\right]^{2}\right\rangle \underset{t_{n} \rightarrow \infty}{\sim} t_{n}^{\alpha} \tau_{n}^{2 / d_{w}-\alpha}=R^{2} .
$$

The $n$ dependence of $t_{n}$ can be then found self-consistently. We assume the scaling

$$
\tau_{n} \underset{n \rightarrow \infty}{\sim} \frac{R^{v}}{n^{\delta}},
$$

where the exponents $\delta$ and $v$ will be determined below. Since $t_{n}=\sum_{k=1}^{n-1} \tau_{k}$, where the variables $\tau_{k}$ have finite mean, we 

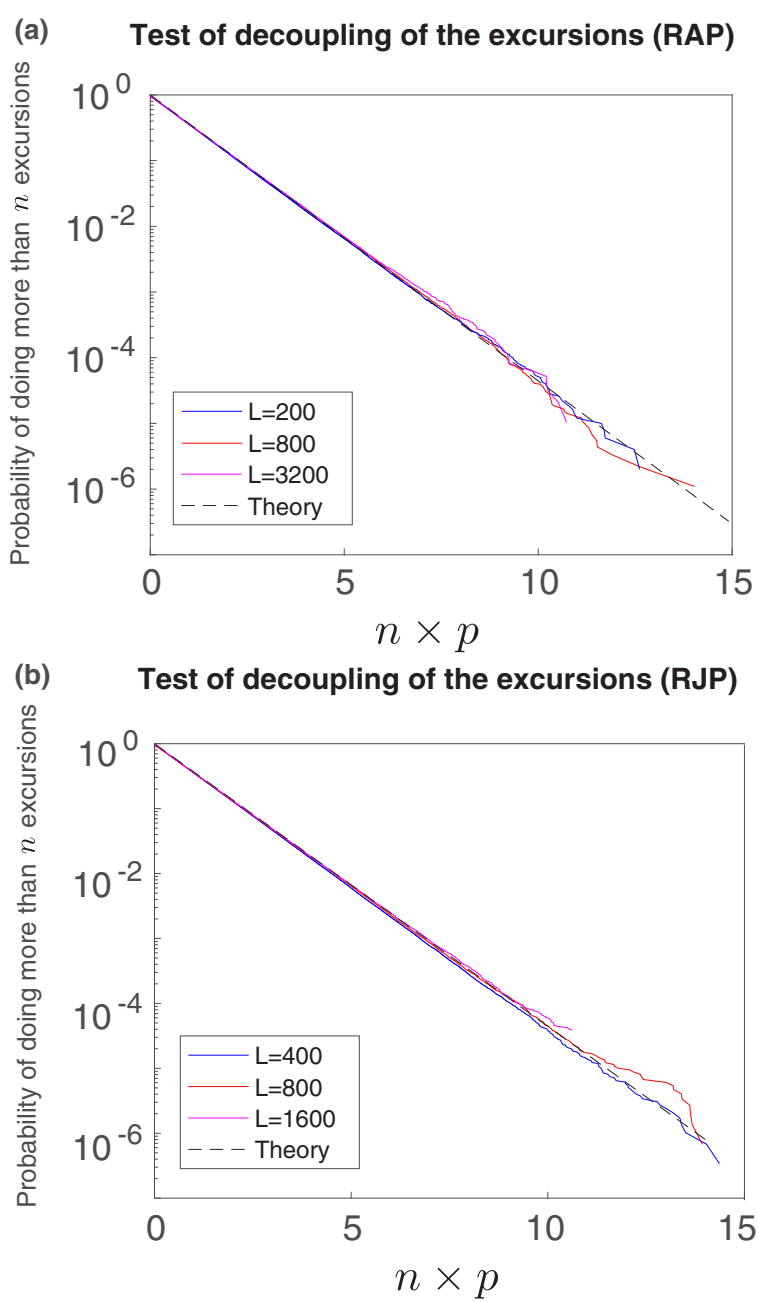

FIG. 5. For both processes, the walker begins at position $r=10$ in a $2 \mathrm{D}$ domain of linear size $R$, with periodic boundary conditions: test of decoupling of the excursions for (a) the RAP and (b) the RJP. Following our prediction, $p$ is taken as $p \propto(a / R)$ for both processes. To compare to (A13), we make use of $(1-p)^{n} \simeq e^{-n p}$ for large $R$.

obtain

$$
t_{n} \underset{n \rightarrow \infty}{\sim} R^{v} n^{1-\delta} .
$$

The values of $\delta$ and $v$ are found by inserting the expressions (A17) and (A18) into Eq. (A16), leading to

$$
\begin{gathered}
v=d_{w}, \\
\delta=\frac{\alpha d_{w}}{2} .
\end{gathered}
$$

Finally,

$$
\begin{gathered}
t_{n} \underset{R \rightarrow \infty}{\sim} R^{d_{w}} n^{1-\alpha d_{w} / 2}, \\
\tau_{n} \underset{R \rightarrow \infty}{\sim} \frac{R^{d_{w}}}{n^{\alpha d_{w} / 2}} .
\end{gathered}
$$

Next we note that $\Phi_{n}(t)$ can be written as

$$
\Phi_{n}(t)=\frac{1}{t} g\left(\frac{t}{t_{n}}, \frac{R^{d_{w}}}{t_{n}}, \frac{a}{R}\right) .
$$

Taking the limits $a / R \rightarrow 0$ and $n \rightarrow \infty$ [which implies that $t_{n} \gg R^{d_{w}}$ from Eq. (A21)] thus leads to the scaling form

$$
\Phi_{n}(t) \sim \frac{1}{t} \phi\left(\frac{t}{t_{n}}\right) .
$$

We check the validity of this scaling in Fig. 6.

The large- $R$ asymptotics of $F(t, a, r, R)$ can be obtained by transforming the discrete sum of Eq. (A13) into an integral

$$
\begin{aligned}
& F(t, a, r, R) \\
& \underset{R \rightarrow \infty}{\sim} p \frac{1-p_{0}}{1-p} \int_{0}^{\infty} \frac{1}{t} \phi\left(\frac{t}{R^{d_{w}} n^{1-\alpha d_{w} / 2}}\right) e^{n \ln (1-p)} d n,
\end{aligned}
$$

which, by using the change of variables $u=n p$ (with $p \ll 1$ ) and Eqs. (A14) and (A15), leads to

$$
\begin{aligned}
F(t, a, r, R) \underset{R \rightarrow \infty}{\sim} & {\left[1-C\left(\frac{a}{r}\right)^{\psi}\right] \frac{1}{t} } \\
& \times \int_{0}^{\infty} \phi\left(\frac{t}{R^{d_{w}\left[u(R / a)^{\psi} / C\right]^{1-\alpha d_{w} / 2}}}\right) \\
& \times e^{-u} d u,
\end{aligned}
$$

where $C$ is a model-dependent numerical constant. A characteristic time appears in Eq. (A26),

$$
T_{\text {typ }}=R^{d_{w}}(R / a)^{\psi\left(1-\alpha d_{w} / 2\right)},
$$

in terms of which the FPT distribution becomes

$$
\begin{aligned}
& F(t, a, r, R) \\
& \underset{R \rightarrow \infty}{\sim}\left[1-C\left(\frac{a}{r}\right)^{\psi}\right] \frac{1}{t} \int_{0}^{\infty} \phi\left(\frac{t}{T_{\mathrm{typ}} u^{1-\alpha d_{w} / 2}}\right) e^{-u} d u .
\end{aligned}
$$

We now consider the rescaled time

$$
\eta \equiv \frac{t}{R^{d_{w}}(R / a)^{\psi\left(1-\alpha d_{w} / 2\right)}}=\frac{t}{T_{\mathrm{typ}}} .
$$

The distribution $G$ of this rescaled variable is given by

$$
G(\eta, r, R)=F(t, a, r, R) T_{\text {typ }}
$$

and we finally obtain that $G$ can be written as

$$
G(\eta, r, R)=\left[1-C\left(\frac{a}{r}\right)^{\psi}\right] h(\eta),
$$

where $h$ is an undetermined scaling function $a$ priori process dependent, which reads here $h(\eta)=$ $\eta^{-1} \int_{0}^{\infty} \phi\left(\eta u^{\left(\alpha d_{w} / 2-1\right)} / C\right) e^{-u} d u$.

Finally, in the marginal case, where $\psi=0$, all the steps presented for the noncompact case hold, with the only difference that now

$$
p \underset{R \gg a}{\sim} \frac{1}{\ln \left(\frac{R}{a}\right)}
$$

and

$$
p_{0} \underset{r \gg a}{\sim} \frac{1}{\ln \left(\frac{r}{a}\right)} .
$$

This leads to the marginal case of Eqs. (1) and (2). 
(a) CDF of the duration of $n$ excursions for BM

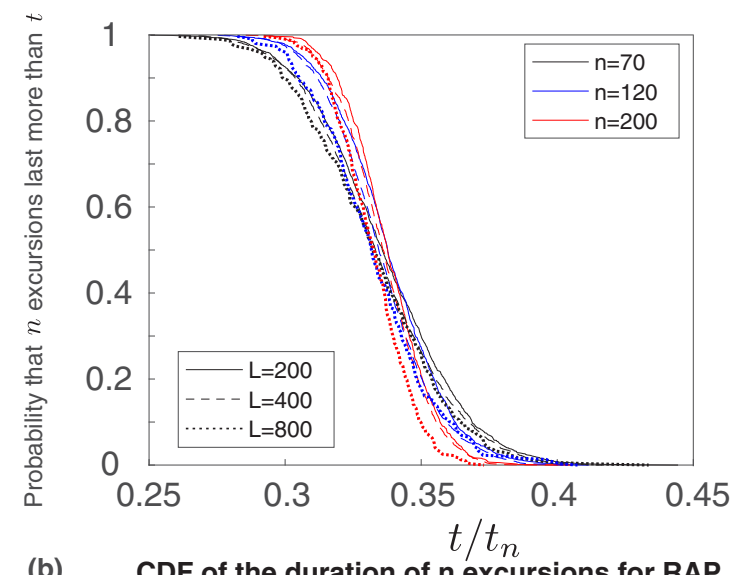

(b) CDF of the duration of $n$ excursions for RAP

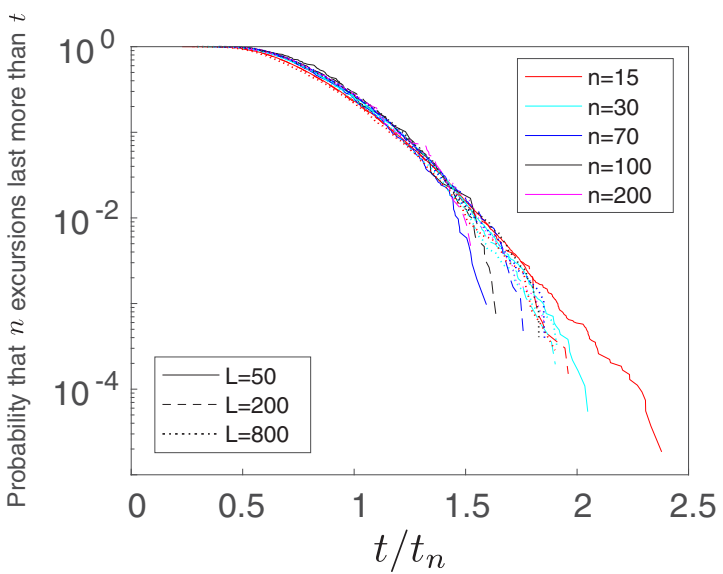

(c) CDF of the duration of $n$ excursions for RJP

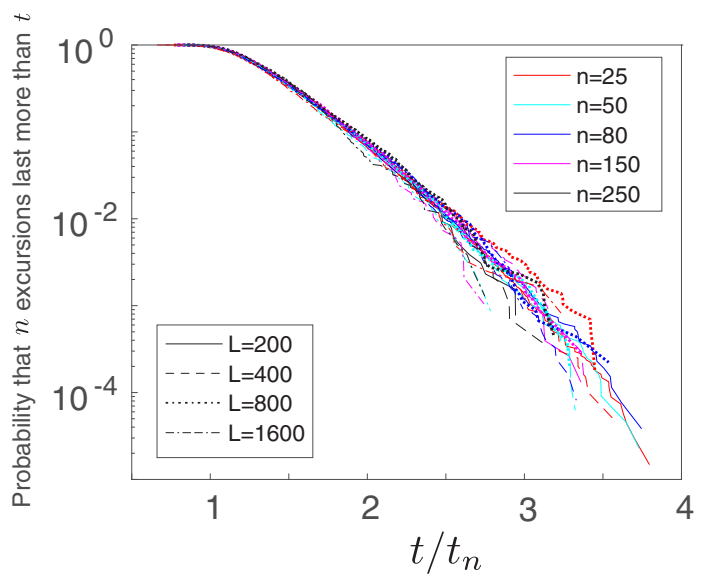

FIG. 6. Validity of the scaling (A24) of the conditional FPT distribution. We tested the scaling of the conditional FPT distribution with the number of excursions $n$ and the size $R$ of the volume. (a) Result for the cumulative distribution function (CDF) of the duration of $n$ excursions for regular Brownian motion. By direct application of the central limit theorem, we know that at large $n$ the distribution $\phi_{n}(t)$ is a Gaussian around its mean (which is proportional to $t_{n} \sim n R^{2}$ ) and variance $\sim \sqrt{n}$. Hence, after rescaling by $t_{n}$, the Heaviside step function is observed. The (b) random acceleration process and (c) random jerk process are also considered. In these accelerating processes, the duration of excursions are shorter and shorter and $t_{n}$ is sublinear with $n$. Our predictions for the scaling with $t_{n}=(n R)^{2 / 3}$ for the RAP and $t_{n}=(n R)^{2 / 5}$ for the RJP are verified.

\section{Modification of the formalism in the case of infinite MFPT}

We describe here how the formalism is modified in the case of processes leading to infinite mean FPTs. This is typically the case of jump processes with broad waiting times, such as CTRWs. For these jumps process, attention must be paid in the computation of $\alpha$. As this exponent defines the potential aging of the duration of excursions (which begin just after a jump), $t$ and $t+\tau$ in the definition of $\alpha$ are taken just after a jump. With this prescription, we find that $\alpha=0$ for a CTRW (note that this contrasts with other definitions of the increments [19], which result in nonzero values of $\alpha$ ).

In the compact case, all steps involved in the proof of Eqs. (1) and (2) hold and therefore these equations are still valid in the case of infinite MFPTs.

The case of noncompact processes requires a separate treatment. We consider the case where the time of the $n$th excursion has a broad distribution, leading to an infinite mean FPT to the target. Specifically, we consider the case where the distribution of $\tau_{n}$ has a power-law tail

$$
P\left(\tau_{n}\right) \sim \frac{\tilde{\tau}^{\gamma}}{\tau_{n}^{1+\gamma}} \frac{1}{n^{\delta}},
$$

with $\gamma \in] 0,1\left[\right.$ and $\tilde{\tau} \propto R^{d_{w}}$. The exponent $\delta$, defined by Eq. (A34), describes the aging of the durations of successive excursions. It is important to note that $t_{n}=\sum_{k=1}^{n-1} \tau_{k}$ is a sum of now broadly distributed random variables, so the scalings (A21) and (A22) are not valid anymore. Here the scaling of $t_{n}$ with $n$ is conveniently found by starting from the Laplace transform

$$
\begin{aligned}
\left\langle e^{-s t_{n}}\right\rangle & =\prod_{i=1}^{n}\left\langle e^{-s \tau_{i}}\right\rangle \\
& =\exp \left(\sum_{i=1}^{n} \ln \left\langle e^{-s \tau_{i}}\right\rangle\right) \\
& =\exp \left[\sum_{i=1}^{n} \ln \left(1-\frac{(s \tilde{\tau})^{\gamma}}{i^{\delta}}+\cdots\right)\right] \\
& =\exp \left[-\left(s \tilde{\tau} n^{(1-\delta /) \gamma}\right)^{\gamma}+\cdots\right],
\end{aligned}
$$

which leads to

$$
t_{n} \underset{R \rightarrow \infty}{\sim} R^{d_{w}} n^{(1-\delta /) \gamma} .
$$

Using Eq. (A16), it is found that here also

$$
\delta=\frac{\alpha d_{w}}{2} .
$$

Finally, inserting the scaling (A36) into Eq. (A13), we find that the FPT distribution is given by Eq. (3) with $\eta=t / T_{\text {typ }}$, but that the typical time is now

$$
T_{\text {typ }}=R^{d_{w}}\left(\frac{R}{a}\right)^{(\psi / \gamma)\left(1-\alpha d_{w} / 2\right)} .
$$

Note that, in the case of CTRWs with infinite mean waiting times, the formula (A48) does not directly apply, because of divergences of both the numerator and denominator of (A45). Since $\psi$ is a purely geometrical exponent, $d_{w}$ in Eq. (A48) has 
to be replaced by the fractal dimension of the trajectory (i.e., the walk dimension of the process without waiting times).

\section{Explicit scalings of the moments of the FPT with geometrical parameters}

The expressions of the distribution $G$ presented in the previous sections allow us to determine the dependence on the geometrical parameters of all the FPT moments (when they exist)

$$
\left\langle T^{m}\right\rangle \sim \begin{cases}R^{d_{w}(m-\theta)} r^{d_{w} \theta} & \text { (compact) } \\ \frac{R^{d_{w}}}{(\ln R / a)^{\alpha d_{w} / 2}} \ln \frac{r}{a} & \text { (marginally compact) } \\ \frac{R^{m\left(d(d)+\psi\left(1-\alpha d_{d} / 2\right)\right)}}{a^{m\left(\psi\left(1\left(1-\alpha d_{w} / 2\right)\right)\right.}}\left[1-C\left(\frac{a}{r}\right)^{\psi}\right] & \text { (noncompact). }\end{cases}
$$

5. Derivation of the formula $\theta=1-d_{f} / d_{w}$ for the persistence exponent of processes with stationary increments (compact case)

We argue here that the relation

$$
\theta=1-\frac{d_{f}}{d_{w}}
$$

holds for general processes with stationary increments. First, this relation holds for Markov processes, as can be shown from a classical renewal equation [37]. Second, it can be recovered by comparing the result

$$
\langle T\rangle \sim R^{d_{w}(1-\theta)}
$$

obtained in the main text for compact processes (and $r$ fixed) and

$$
\langle T\rangle \sim R^{d_{f}}
$$

obtained in [9] for non-Markovian Gaussian processes with stationary increments. Note that the results of [9] are exact perturbatively at order $\epsilon^{2}$ around Markovian processes and quantitatively accurate even for strongly non-Markovian processes. Finally, for FBM in one dimension, it was obtained by scaling arguments in [12] and shown mathematically in [14].

\section{Transience exponent $\psi$ (noncompact case): Derivation of Eq. (4)}

\section{a. Processes with stationary increments at long times}

i. Markovian processes (stationary increments). For Markovian processes, the transience exponent $\psi$ can be obtained from the renewal equation. It relates the propagator $P\left(\mathbf{r}, t \mid \mathbf{r}^{\prime}\right)$ (the probability density that the walker is at $\mathbf{r}$ at $t$ starting from $\mathbf{r}^{\prime}$ at $t=0$ ) and the first-passage time density $F\left(t \mid \mathbf{r}^{\prime}\right)$ to reach the spherical target of radius $a$ centered at $\mathbf{0}$ at time $t$,

$$
P(\mathbf{0}, t \mid \mathbf{r})=\int_{0}^{t} d t^{\prime} F\left(t^{\prime} \mid \mathbf{r}\right) P\left(\mathbf{0}, t \mid a, t^{\prime}\right),
$$

where $P\left(\mathbf{0}, t \mid a, t^{\prime}\right)$ is the propagator averaged over the starting points on the sphere of radius $a$ centered at $\mathbf{0}$. For a process that has stationary increments, one can write $P\left(\mathbf{0}, t \mid a, t^{\prime}\right)$ as a function of the time lag $t-t^{\prime}$ only,

$$
P(\mathbf{0}, t \mid \mathbf{r})=\int_{0}^{t} d t^{\prime} F\left(t^{\prime} \mid \mathbf{r}\right) P\left(\mathbf{0}, t-t^{\prime} \mid a\right) .
$$

Integrating this equation over time $t$ from 0 to $\infty$, we obtain the following expression of the probability $p$ to eventually reach the target:

$$
p=\frac{\int_{0}^{\infty} d t P(\mathbf{0}, t \mid \mathbf{r})}{\int_{0}^{\infty} d t P(\mathbf{0}, t \mid a)}
$$

Using scale invariance, the propagator can be written as [38]

$$
P(\mathbf{0}, t \mid \mathbf{r}) \sim \frac{1}{t^{d_{f} / d_{w}}} \Pi\left(\frac{r}{t^{1 / d_{w}}}\right)
$$

and we finally obtain

$$
p \sim\left(\frac{a}{r}\right)^{d_{f}-d_{w}}
$$

i.e.,

$$
\psi=d_{f}-d_{w} .
$$

ii. Non-Markovian Gaussian processes with stationary increments. For general non-Markovian processes, the exponent $\psi$ has not been studied and no exact results are available. However, by comparing the result

$$
\langle T\rangle \sim R^{d_{w}+\psi}
$$

obtained in the main text for noncompact processes (and $r$ fixed) and

$$
\langle T\rangle \sim R^{d_{f}}
$$

obtained in [9] for non-Markovian Gaussian processes with stationary increments, we obtain again

$$
\psi=d_{f}-d_{w}
$$

for non-Markovian Gaussian processes with stationary increments. Note that, from the very definition of $\psi$ (which involves the small-target-size limit), this argument can be extended to processes whose increments are only asymptotically stationary. In addition, we expect that the validity of this result is broader than the case of Gaussian processes.

\section{b. Processes with nonzero aging exponent $\alpha$}

We now consider a process with nonzero aging exponent $\alpha$ such that

$$
\left\langle[X(t+\tau)-X(t)]^{2}\right\rangle \underset{t \rightarrow \infty}{\sim} t^{\alpha} \tau^{2 / d_{w}-\alpha} .
$$

Let us define the time-changed process

$$
X^{*}(t) \equiv X\left(t^{b}\right)
$$

Its increments satisfy, on the one hand,

$$
\begin{aligned}
\left\langle\left[X^{*}(t+\tau)-X^{*}(t)\right]^{2}\right\rangle & =\left\langle\left[X\left((t+\tau)^{b}\right)-X\left(t^{b}\right)\right]^{2}\right\rangle \\
& \sim t^{\alpha b}\left((t+\tau)^{b}-t^{b}\right)^{2 / d_{w}-\alpha} \\
& \sim t^{b \alpha+(b-1)\left(2 / d_{w}-\alpha\right)} \tau^{2 / d_{w}-\alpha},
\end{aligned}
$$

and on the other hand, by definition,

$$
\left\langle\left[X^{*}(t+\tau)-X^{*}(t)\right]^{2}\right\rangle \underset{t \rightarrow \infty}{\sim} t^{\alpha^{*}} \tau^{2 / d_{w}^{*}-\alpha^{*}} .
$$

By identification, this leads to

$$
\alpha^{*}=\alpha+(b-1) \frac{2}{d_{w}}
$$


and

$$
d_{w}^{*}=\frac{d_{w}}{b} .
$$

This shows that the process $X^{*}$ has asymptotically stationary increments if we choose $b$ with the value

$$
b=1-\frac{\alpha d_{w}}{2} \text {. }
$$

The analysis of the preceding section then applies to $X^{*}(t)$ and yields

$$
\psi_{X^{*}}=d_{f}-d_{w}^{*}=d_{f}-\frac{d_{w}}{1-\frac{\alpha d_{w}}{2}} .
$$

From the definition of $\psi$, it is clear that this exponent is invariant under a generic clock change of the process. As a result,

$$
\psi_{X}=\psi_{X^{*}}=d_{f}-\frac{d_{w}}{1-\frac{\alpha d_{w}}{2}},
$$

where we have used the result of Appendix A 6 a on processes with stationary increments at long times.

\section{APPENDIX B: EXPLICIT RESULTS FOR SPECIFIC STOCHASTIC PROCESSES AND DETAILS OF NUMERICAL SIMULATIONS}

In this Appendix we consider several examples of stochastic processes, for which we determine the aging exponent $\alpha$ and the persistence exponent $\theta$, and deduce the FPT distribution in confinement. We also describe the algorithms used to perform the simulations of these stochastic processes, as shown in Figs. 2-4.

\section{Processes with stationary increments (compact case)}

In the case of compact processes with stationary increments, according to Eq. (2), the distribution of the rescaled variable $\eta=T / R^{d_{w}}$, in the large- $R$ limit, is

$$
G(\eta ; a, r, R)=h(\eta)\left(\frac{r}{R}\right)^{d_{f}-d_{w}},
$$

where we have used the relation $\theta=1-\frac{d_{f}}{d_{w}}$ valid for processes with stationary increments (see Appendix A 5). When the moments of the FPT exist, they are thus given by

$$
\left\langle T^{m}\right\rangle \sim R^{d_{w}(m-1)+d_{f}} r^{d_{w}-d_{f}} .
$$

As a particular case of this general expression, we recover the scalings with $r$ and $R$ of the case of scale-invariant Markovian processes (stationary increments) (see Ref. [8] for the first moment and Ref. [17] for the full distribution) of the mean FPT $\langle T\rangle$ in the case of a one-dimensional fractional Brownian motion (which is a scale-invariant Gaussian non-Markovian process with stationary increments).

\section{Processes with stationary increments at long times (noncompact case)}

As stated in the main text, in the noncompact case, the FPT distribution depends only on $d_{f}, d_{w}$, and $\alpha$. In turn, the dynamical exponents $d_{w}$ and $\alpha$ depend only on the long-time asymptotics of the process.

In the case of processes with stationary increments at long times (i.e., $\alpha=0$ ), we have, from Appendix A 6 a,

$$
\psi=d_{f}-d_{w} .
$$

The rescaled variable is thus

$$
\eta \equiv \frac{T a^{d_{f}-d_{w}}}{R^{d_{f}}}
$$

and its distribution in the large- $R$ limit is given by

$$
G(\eta ; a, r, R)=h(\eta)\left[1-C\left(\frac{a}{r}\right)^{d_{f}-d_{w}}\right] .
$$

When the moments of the FPT exist, they are thus given by

$$
\left\langle T^{m}\right\rangle \sim\left(\frac{R^{d_{f}}}{a^{d_{f}-d_{w}}}\right)^{m}\left[1-C\left(\frac{a}{r}\right)^{d_{f}-d_{w}}\right] .
$$

As particular cases of this general expression, we recover the scalings with $r$ and $R$ of scale-invariant Markovian processes (see [8] for the first moment and [17] for the full distribution).

Note that these scalings hold in the important case of a $d$ dimensional fractional Brownian motion (for both equilibrated and nonequilibrated initial conditions) such that $H<1 / d$, with $d_{f}=d$ (in order to have a noncompact process) (also discussed below).

\section{Fractional Brownian motion with equilibrated or nonequilibrated initial conditions}

\section{a. Theoretical results}

The 1D FBM is the Gaussian process with constant mean (here we set this mean to $X_{0}$ ) and correlations $\langle[X(t)-$ $\left.\left.X_{0}\right]\left[X\left(t^{\prime}\right)-X_{0}\right]\right\rangle=K\left[t^{2 H}+t^{2 H}-\left|t-t^{\prime}\right|^{2 H}\right]$. Here $H$ is the Hurst exponent $(0<H<1)$ and we take $K=1$. We define the $d$-dimensional FBM as $\mathbf{X}(t)=\left(X_{1}(t), \ldots, X_{d}(t)\right)$, where the $X_{i}(t)$ are independent one-dimensional FBMs. The FBM is a non-Markovian process with stationary increments; the results of the former sections therefore apply with a walk dimension $d_{w}=1 / H$ : The FPT statistics in a large confining volume follow Eqs. (B1) and (B2) in the compact case $(d<1 / H)$ and Eqs. (B5) and (B6) in the noncompact case $(d>1 / H)$. We tested both cases in our simulations (see below for details).

Since $X(t)$ is a non-Markovian process, its statistics depend on the one of trajectories in the past $(t<0)$. To specify the initial state, it is useful to consider a microscopic model which is equivalent to a FBM in specific limits. We introduce the stochastic process $X(t)$ defined as the local height of a fluctuating interface $h(x, t)$ at a given position $X(t)=h\left(x_{0}, t\right)$. Following Ref. [12], we assume the dynamics

$$
\frac{\partial h}{\partial t}=-\left(-\nabla^{2}\right)^{1 /(2-4 H)} h+\xi(x, t),
$$

where $\xi(x, t)$ is a Gaussian white noise, satisfying $\left\langle\xi(x, t) \xi\left(x^{\prime}, t^{\prime}\right)\right\rangle=\delta\left(t-t^{\prime}\right) \delta\left(x-x^{\prime}\right)$. Then $\quad X(t)$ is a Gaussian process. It has been shown that the persistence exponent of $X(t)$ is then strongly dependent on the initial interface height distribution [12]. 
If the interface is initially equilibrated, then $\left\langle X(t) X\left(t^{\prime}\right)\right\rangle=$ $t^{2 H}+t^{2 H}-\left|t-t^{\prime}\right|^{2 H}$ and $X(t)$ is a fractional Brownian motion with Hurst exponent $H$ and has stationary increments. If the interface is initially flat, $h(x, t=0)=0$ for all $x$, then the process $X(t)$ becomes non-Markovian with nonstationary increments and the correlator reads

$$
\left\langle X(t) X\left(t^{\prime}\right)\right\rangle=\left(t+t^{\prime}\right)^{2 H}-\left|t-t^{\prime}\right|^{2 H} .
$$

In this case, we call the process $X(t)$ an initially quenched fractional Brownian motion. Its exponent $\theta$ is not known analytically, but can be determined numerically. In Fig. 2(a), Eq. (4) for the mean FPT is checked in the particular case $H=3 / 8$, in which case one can approximate $\theta(H=3 / 8)=$ $0.84 \ldots$ [12]. In this case, $\langle T\rangle$ is sublinear in $V$. In order to obtain an example of process with $\langle T\rangle$ superlinear in $V$, we consider the process defined by Eq. (B8) with $H=0.65$. In this case, $\theta \simeq 0.3$ (see Ref. [12]). Note that this process cannot be easily matched on the evolution of a point of an interface, contrarily to the case $H=3 / 8$.

\section{b. Description of simulations}

i. One-dimensional FBM. The algorithm used to sample the 1D FBM trajectories is the circulant matrix algorithm (also called the Davies-Harte method) [39-41]. This method generates trajectories $X\left(t_{i}\right)$ with a constant time step $\Delta t=$ $t_{i+1}-t_{i}$, until a fixed maximal time $t_{\max }$, with a number of operations of the order of $N \ln N$, with $N=t_{\max } / \Delta t$. The value of $t_{\max }$ is chosen so that the number of trajectories that do not reach the target is negligible. The time step $\Delta t$ is then reduced until convergence is obtained, with a FPT density that does not depend on $\Delta t$. In Figs. 2(a) and 3(a), for $H=0.4$, we use $t_{\max }=524888$ and $\Delta t=0.1$, except for $R=320$, where $t_{\max }$ is doubled, with the same time step. The initial position is set to $X_{0}=1$. It was checked in Ref. [9] that defining reflecting boundaries with the Hosking algorithm does not change the mean FPT in the large-volume limit. In Fig. 2(a), the mean FPT and the volume $V=R$ are rescaled by the factors $\left(V_{0} ; T_{0}\right)=(20 ; 27.2)$.

ii. Two-dimensional FBM. The two-dimensional FBM is defined as $\mathbf{X}=\left(X_{1}(t), X_{2}(t)\right)$, where the $X_{1}(t)$ and $X_{2}(t)$ are one-dimensional independent FBMs. Further, $X_{1}(t)$ and $X_{2}(t)$ are simulated with the circulant matrix algorithm described above. The target radius is set to $a=1$ and the domain defined as the square of size $\sqrt{\pi} R$ with periodic boundary conditions. In Fig. 3(b), for $H=0.35$, we use $t_{\max }=2 \times 10^{6}$ and a time step $\Delta t=0.1$. In Figs. 3(d) and 2(b), for $H=0.7$, we use $t_{\max }=10^{6}$ and $\Delta t=0.1$ and the initial distance to the target is $r=10$. In Fig. 2(b), the results are rescaled by the factors $\left(V_{0} ; T_{0}\right)=(50 ; 155.4)$.

iii. One-dimensional FBM with nonequilibrated initial conditions and $H=3 / 8$. We consider here the process defined as $X(t)=h(0, t)$, where $h(x, t)$ is the height of an interface. As discussed in Appendix B 3, $X(t)$ is a FBM only when the initial distribution of $h(x, t)$ is the equilibrium distribution. Here we consider that the interface is initially flat, $h(x, t=$ $0)=X_{0}$. In Fig. 2(a), $X_{0}=10$. The simulations are performed by the stochastic integration of the Langevin equation (B7) following the algorithm of Ref. [12], with $H=3 / 8$. The interface is described by its height at a finite number $N$

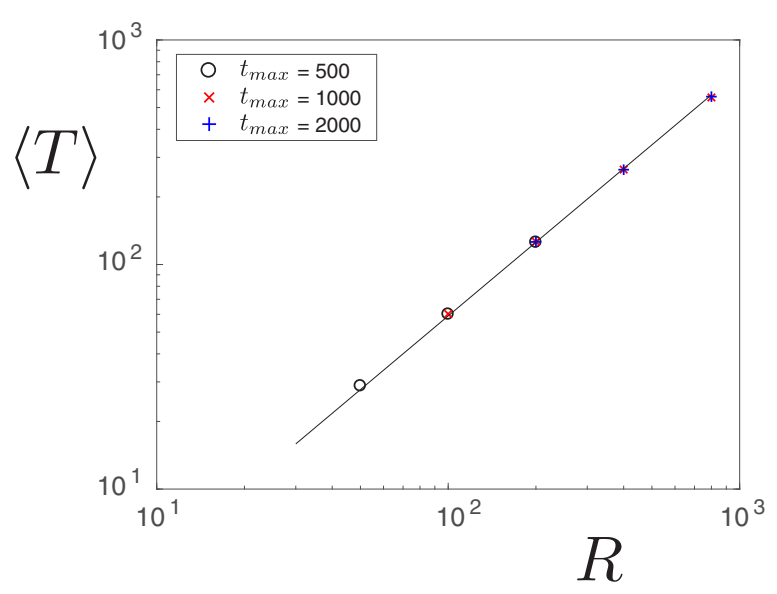

FIG. 7. Control of the validity of the estimator (B9) for the MFPT in simulations of the 1D initially quenched FBM, with $H=0.65$ (see the text).

of positions, $h_{i}(t)=h(i \Delta x, t)$. Here we take $\Delta x=1$ and $N=200$ discrete positions and we use periodic boundary conditions $\left(h_{0}=h_{N}\right)$. The time step is set to $\Delta t=0.1$ and the convergence of the results with the time step is checked. The maximal time is $t_{\max }=4 \times 10^{7}$. It is checked that the relaxation time of the system (which grows as $N^{4}$ for $H=3 / 8$ ) is much larger than $t_{\max }$. In Fig. 2(a), the results are rescaled by the factors $\left(V_{0} ; T_{0}\right)=(160 ; 91.3)$.

iv. One-dimensional initially quenched FBM $H=0.65$. We consider here the simulations of a process $X(t)$ starting at $X_{0}=$ $r=1$ with covariance given by Eq. (B8). The targets are at the positions 0 and $R$. We simulate the trajectories at $N$ discrete times $t_{n}=n \Delta t$ by using a Cholesky decomposition of the covariance matrix $\Sigma_{i j} \equiv\left\langle X\left(t_{i}\right) X\left(t_{j}\right)\right\rangle$, which can be written as $\Sigma=L L^{t}$, with $L$ a lower triangular matrix. Then a trajectory is generated by computing $X\left(t_{i}\right)=\sum_{j=1}^{N} L_{i j} u_{j}$, where the $u_{j}$ are independent Gaussian variables of zero mean and variance unity. The time step is set to $\Delta t=0.03$ to ensure convergence. Because of the large memory requirement of this algorithm (it increases as $N^{2}$ ), we could not choose $t_{\max }=N \Delta t$ large enough to obtain a negligible proportion $q$ of trajectories which do not find the target. In order to avoid errors in the measurement of the MFPT, we proceed as follows. Observing that the tail of the FPT distribution is exponential, we fit the tail of the survival probability with $S(t) \sim B(R / r)^{\theta / H} e^{-a t / R^{1 / H}}$. Then, calling $T_{\text {emp }}$ the MFPT restricted to successful runs (that is, with a FPT smaller than $\left.t_{\max }\right)$, the MFPT is estimated with the formula

$$
\begin{aligned}
\langle T\rangle= & (1-q) T_{\mathrm{emp}}+a B R^{(\theta-1 / H} e^{-a t_{\max } / R^{1 / H}} \\
& \times\left(1+a t_{\max } / R^{1 / H}\right)
\end{aligned}
$$

We check the validity of this procedure by ensuring that different $t_{\max }$ lead to the same result for the MFPT (see Fig. 7). In Fig. 2(a), the results are rescaled by the factors $\left(V_{0} ; T_{0}\right)=(50 ; 28.8)$. In Fig. 3(c), we use $t_{\max }=500$ for $R=50, t_{\max }=1000$ for $R=100,200$, and $t_{\max }=2000$ for $R=400$. 


\section{Random acceleration process and its generalizations}

\section{a. Theoretical results}

We consider here the $d$-dimensional process $\mathbf{X}(t)=$ $\left(X_{1}(t), \ldots, X_{d}(t)\right)$ of order $n(n \geqslant 2)$, defined by

$$
\frac{d^{n}}{d t^{n}} X_{i}(t)=\xi_{i}(t)
$$

where $\xi_{i}(t)$ is a Gaussian white noise with zero mean satisfying $\left\langle\xi_{i}(t) \xi_{j}\left(t^{\prime}\right)\right\rangle=K \delta\left(t-t^{\prime}\right) \delta_{i j}$, with $K$ a coefficient that can be set to unity by appropriate rescaling, $K=1$. Then $\mathbf{X}(t)$ is a non-Markovian process, with nonstationary increments. For $n=2, X_{i}(t)$ is a random acceleration process; for $n>2$ one obtains higher-order processes. We will consider in particular the case $n=3$ (random jerk process).

We first investigate the aging features of this class of processes. Denoting by $x$ a generic coordinate $X_{i}$ and using the representation $x(t)=\int_{0}^{t}\left|t-t^{\prime}\right|^{n-1} \xi\left(t^{\prime}\right) d t^{\prime}$, we compute easily the autocorrelation function

$$
\begin{aligned}
& \langle x(t+\tau) x(t)\rangle \\
& =\int_{0}^{t+\tau} d t_{1} \int_{0}^{t} d t_{2}\left|t+\tau-t_{1}\right|^{n-1}\left|t-t_{2}\right|^{n-1} \delta\left(t_{1}-t_{2}\right) \\
& =t^{2 n-1} \int_{0}^{1} x^{n-1}[x+(\tau / t)]^{n-1} d x .
\end{aligned}
$$

Hence the increments are given by

$$
\begin{aligned}
& \left\langle[x(t+\tau)-x(t)]^{2}\right\rangle \\
& =(t+\tau)^{2 n-1} \int_{0}^{1} x^{2 n-2} d x-2 t^{2 n-1} \\
& \quad \times \int_{0}^{1} x^{n-1}[x+(\tau / t)]^{n-1} d x+t^{2 n-1} \int_{0}^{1} x^{2 n-2} d x .
\end{aligned}
$$

It is straightforward to see that both terms proportional to $t^{2 n-1}$ and $t^{2 n-2}$ do cancel. Finally, we get

$$
\left\langle[x(t+\tau)-x(t)]^{2}\right\rangle \propto t^{2 n-3} \tau^{2} .
$$

The walk dimension $d_{w}$ and the aging exponent $\alpha$ are then given by (for $n \geqslant 2$ )

$$
d_{w}(n)=\frac{2}{2 n-1}, \quad \alpha(n)=2 n-3,
$$

where we have used the definition (see the main text)

$$
\left\langle[X(t+\tau)-X(t)]^{2}\right\rangle \sim_{t \rightarrow \infty} t^{\alpha} \tau^{2 / d_{w}-\alpha} .
$$

As a consequence, the transience exponent is

$$
\psi=d-1 .
$$

For $d>1, \psi>0$ and the process is therefore noncompact, whereas it is compact if $d=1$.

Our theoretical results then predict that

$$
T_{\mathrm{typ}}= \begin{cases}R^{2 /(2 n-1)} & (d=1) \\ R^{2 /(2 n-1)}\left(\frac{R}{a}\right)^{2(d-1) /(2 n-1)} & (d>1)\end{cases}
$$

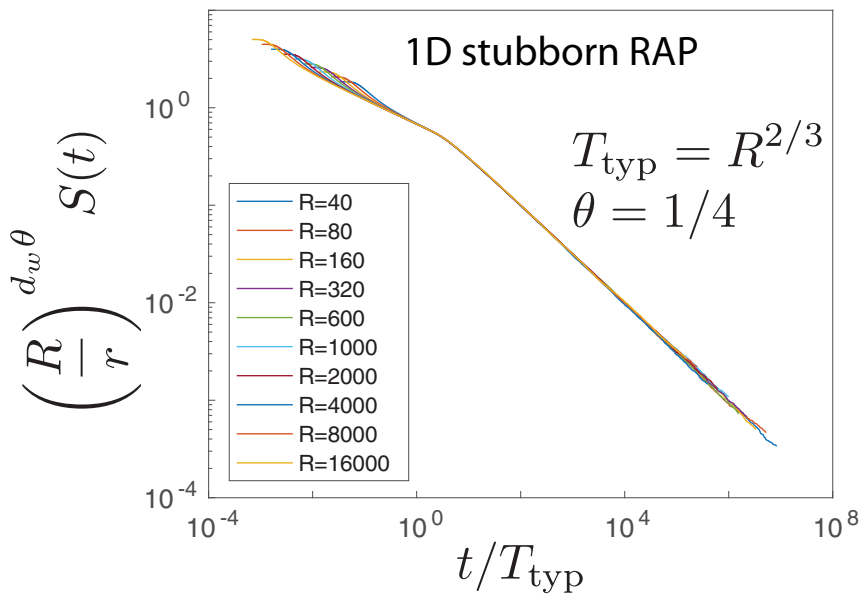

FIG. 8. Validity of the scalings of the FPT distribution with geometrical parameters for the stubborn RAP.

and $\eta=T / T_{\text {typ }}$ is asymptotically distributed in the limit of large volume according to

$$
G(\eta ; a, r, R)= \begin{cases}h(\eta)\left(\frac{r}{R}\right)^{2 \theta /(2 n-1)} & (d=1) \\ h(\eta)\left[1-C\left(\frac{a}{r}\right)^{d-1}\right] & (d>1) .\end{cases}
$$

In the compact case $d=1$, the persistence exponent is known either exactly (for $n=2$ ) or approximately (for $n \geqslant 3$ ). It is also known in the case of a partially absorbing target, where the random walker has a finite probability $q$ to actually find the target at each visit

$$
\theta= \begin{cases}1 / 4 & (n=2) \\ 0.2202 \ldots & (n=3) \\ \frac{1}{4}\left[1-\frac{6}{\pi} \sin ^{-1}\left(\frac{1-q}{2}\right)\right] & (n=2, \text { finite } q) .\end{cases}
$$

Hence, the mean FPT obeys the scaling (when it exists)

$$
\langle T\rangle \sim \begin{cases}R^{2(1-\theta) /(2 n-1)} r^{2(\theta) /(2 n-1)} & (d=1) \\ \frac{R^{2 d /(2 n-1)}}{a^{2(d-1) /(2 n-1)}}\left[1-C\left(\frac{a}{r}\right)^{d-1}\right] & (d>1)\end{cases}
$$

and the higher moments can be obtained by using Eq. (A39). Note that, in particular, in the case of $n=2$ and $d=1$, one has $\langle T\rangle \sim r^{1 / 6} R^{1 / 2}$, which is in agreement with Ref. [42] in the case of reflecting boundary conditions (where the sign of the velocity is immediately reversed when the confining boundary is reached $v \rightarrow-v$ ). We also considered another type of boundary condition, with a confining boundary that stops the particle while its target velocity keeps evolving according to Eq. (B10) until it changes sign and the particle escapes from the boundary. We call the RAP with this kind of boundary conditions the stubborn random acceleration process. In this case the moments of the FPT distributions are infinite, but the distribution of FPT still satisfies the scaling (B18) (Fig. 8).

\section{b. Simulation details}

We now describe the simulations of the random acceleration process and its generalizations that were used in Figs. 2-4.

$i$. Simulations of the random acceleration process $(n=$ 2). The stochastic trajectories are generated by integrating numerically the Langevin equation (B10) with the algorithm introduced in [43]. This algorithm does not generate any error 
due to the time discretization, as it creates the exact probability density function for the joint density position and velocity. We need to choose however a time step $\Delta t$ that is small enough to ensure that the target is not missed during one step. Since the velocity typically grows as $\sqrt{t}$, we have to reduce the time step at each iteration, in order to keep $\Delta x \sim v \Delta t$ small. A simple way to do that is to take the time step of the $n$th step to be $(\Delta t)_{n}=D / n^{1 / 3}$, with a small enough $D$. With this choice, the spreading of the velocity does not increase with $n$. The value of $D$ is decreased up to reach convergence of the results. We choose $D=0.7$ in all cases. The initial velocity is $v_{0}=0$. The following are additional details.

(a) In the case of reflecting conditions in one dimension, the reflecting boundary, located at $x=R / 2$, can be replaced by a second target at $x=R$. The initial position is set to $X_{0}=1$. The probability $q$ of being absorbed at each target crossing event is set to $q=1$ or $q=0.7$. In Fig. 2(a), the mean FPT and the volume $V=R$ are rescaled by the factors $\left(V_{0} ; T_{0}\right)=$ $(40 ; 8.05)$ in the case $q=1$ and $\left(V_{0} ; T_{0}\right)=(20 ; 8.79)$ when $q=0.7$.

(b) In one dimension, we also considered the stubborn acceleration process; when reaching the confining boundary, the velocity at step $i$ evolves according to $v_{i+1}=v_{i}+\sqrt{(\Delta t)_{i}}$, while the position remains constant at $x_{i}=R / 2$ until reaching a negative velocity that drives the walker away from the boundary. The results for this process are presented in Fig. 8.

(c) In two (three) dimensions, we consider a square (cubic) confining volume $V=R^{d}$ which, for reflecting boundary conditions, can be replaced by an infinite periodic array of targets. The initial distance to the target is taken to be $r=10$ and the target radius is $a=1$. In Fig. 2(b), the mean FPT and the volume $V$ are rescaled by the factors $\left(V_{0} ; T_{0}\right)=(100 ; 556.4)$ (in two dimensions) and $\left(V_{0} ; T_{0}\right)=(100 ; 14870)$ (in three dimensions).

ii. Simulations of the random jerk process $(n=3)$. The random jerk process is defined by Eq. (B10), with $n=3$. The trajectories are sampled by using $X\left(t_{n+1}\right)=X\left(t_{n}\right)+$ $Y\left(t_{n}\right)\left(t_{n+1}-t_{n}\right)$, where $Y_{n}$ is a random acceleration process (discussed above). In order to keep the velocity small, we choose $(\Delta t)_{n}=D / n^{3 / 5}$ and we reach convergence for $D=$ 0.1 . In one dimension, the initial distance to the target is $X_{0}=1$ and the domain size $R / 2$. In two dimensions, the initial distance to the target is $r=10$, the target radius is $a=1$, and the domain is a square of size $L R \times R$ with period boundary conditions. In Fig. 2, the mean FPT and the volume $V$ are rescaled by the factors $\left(V_{0} ; T_{0}\right)=(160 ; 7.6)$ [in one dimension, Fig. $\left.2(\mathrm{a})\right]$ and $\left(V_{0} ; T_{0}\right)=(40 ; 24.7)$ [in two dimensions, Fig. 2(b)].

\section{Case of Lévy flights}

We now consider the case of Lévy flights, where at each step a $d$-dimensional walker performs in a random direction a jump whose length $l$ is drawn from a distribution with the power-law tail

$$
p(l) \sim \frac{1}{l^{1+\beta}} .
$$

These processes have been repeatedly invoked in the literature on random search processes [44]. Two definitions of the firstpassage time to the target have been used in previous works.
In the first definition, the target can be detected only when the walker changes direction. This first-passage time will be termed here a first-arrival time [36,45]. In the second definition, the target can be detected as soon as it is crossed by the trajectory of the walker. This first-passage time will be termed here a first-crossing time.

We consider only the case $0<\beta<2$ (note that for $\beta>2$, the process converges to Brownian motion). The dimension of the walk is given by

$$
d_{w}=\beta \text {. }
$$

\section{a. First arrival case}

In this case, the renewal type argument of Appendix A 5 holds. For integer dimensions, the process is compact for $d=1$ and noncompact for $d \geqslant 2$. In the compact case, we have

$$
\theta=1-\frac{d_{f}}{d_{w}}=1-\frac{1}{\beta}
$$

and the aging exponent is 0 . Thus, we predict that the typical time is, for any $\beta \in] 0,2[$,

$$
T_{\mathrm{typ}}= \begin{cases}R^{\beta} & (d=1) \\ \frac{R^{d}}{a^{d-\beta}} & (d=2,3)\end{cases}
$$

and that the distribution of the rescaled FPT $\eta=T / T_{\text {typ }}$ is asymptotically distributed according to

$$
G(\eta ; a, r, R)= \begin{cases}h(\eta)(r / R)^{\beta-1} & (d=1) \\ h(\eta)\left[1-C\left(\frac{a}{r}\right)^{d_{f}-\beta}\right] & (d=2,3 \ldots)\end{cases}
$$

The results of Appendix B 1 apply with $d_{f}=1$ and $d_{w}=\beta$. They are consistent with the Markovian prediction of Ref. [17].

\section{b. First crossing}

In this case, the renewal-type argument of Appendix A 5 does not apply directly. In one dimension, the process is compact and the persistence exponent can be obtained from the spare Anderson theorem $[15,46]$

$$
\theta=1 / 2 .
$$

The rescaled variable $\eta=T / R^{\beta}$ is thus asymptotically distributed in the large- $R$ limit according to the distribution

$$
G(\eta ; a, r, R)=h(\eta)\left(\frac{r}{R}\right)^{\beta / 2}
$$

The moments are then given by

$$
\left\langle T^{m}\right\rangle=R^{\beta(m-1 / 2)} r^{\beta / 2} .
$$

Note that the scalings with $r$ and $R$ of the first moment obtained in [47] are recovered from this general expression of the moments.

In the noncompact case, when $\beta \in] 1,2\left[, d_{w}=\beta\right.$ and $\psi=$ $d_{f}-\beta$. Thus, in this case, the scaling results are the same for both arrival and crossing prescriptions (B25).

The situation is different for $\beta \in] 0,1[$. In this case, the trajectories are the same as for a Lévy walk, for which it is argued below that $\psi=d_{f}-1$. Note that Eq. (3) applies 
for first-passage problems and not directly to first-crossing problems. Finally,

$$
T_{\mathrm{typ}} \sim \frac{R^{d_{f}+\beta-1}}{a^{d_{f}-1}}
$$

and

$$
G(\eta ; a, r, R)=h(\eta)\left[1-C\left(\frac{a}{r}\right)^{d_{f}-1}\right] .
$$

Notably, in this case, the scaling results are therefore different for arrival and crossing prescriptions.

\section{Case of Lévy walks}

Lévy walks provide a natural physical generalization of Lévy flights in which the instantaneous velocity of the walker is bounded, as opposed to Lévy flights. We set here this instantaneous velocity to unity. A $d$-dimensional Lévy walker performs a series of independent and randomly oriented ballistic excursions at constant speed, whose length $l$ is drawn from a distribution with a power-law tail

$$
p(l) \sim \frac{1}{l^{1+\beta}} .
$$

For $\beta \in] 0,1$ [, the process is known to be scale invariant [48], so our results for the FPT apply directly. For $\beta \in] 1,2[$, even if the process is not scale invariant [48], it is known that the bulk of the propagator is scale invariant. Knowing that the weight of the ballistic fronts that compose the tail of the distribution is negligible, these tails are irrelevant for the determination of the FPT statistics and we therefore make use of the scalings of the bulk.

Let us first consider the compact situation, obtained for a one-dimensional case. For $\beta \in] 0,1\left[, d_{w}=1\right.$ and $\theta=\beta / 2$ (see Refs. [15,49]), while for $\beta \in] 1,2\left[, d_{w}=\beta\right.$ (see Ref. [48]) and $\theta=1 / 2$. The typical time is therefore

$$
T_{\mathrm{typ}}= \begin{cases}R & (0<\beta<1, d=1) \\ R^{\beta} & (1 \leqslant \beta<2, d=1)\end{cases}
$$

and for $\beta \in] 0,2[$ the rescaled variable $\eta=T / R$ is asymptotically distributed in the large- $R$ limit according to the distribution

$$
G(\eta ; a, r, R)=h(\eta)\left(\frac{r}{R}\right)^{\beta / 2} .
$$

In the noncompact case, for $\beta \in] 1,2\left[, d_{w}=\beta\right.$ (see Ref. [48]) and $\psi=d_{f}-\beta$. In this case,

$$
T_{\text {typ }} \sim \frac{R^{d_{f}}}{a^{d_{f}-\beta}}
$$

and

$$
G(\eta ; a, r, R)=h(\eta)\left[1-C\left(\frac{a}{r}\right)^{d_{f}-\beta}\right] .
$$

Next, for $\beta \in] 0,1\left[, d_{w}=1\right.$ (see Ref. [48]) and $\psi=d_{f}-1$. In this case, the scaling of $T_{\text {typ }}$ actually depends on the nature of the possible directions of the velocity of Lévy walks. We consider here two natural choices, as defined in Ref. [50]: (i) In the $X Y$ model, the particle is allowed to move only on one axis at a time (implying in particular that the walker in confinement can be trapped in long-lasting periodic trajectories), and (ii) in the uniform model, at each reorientation point the particle chooses a random direction of motion specified by an angle uniformly distributed in $[0,2 \pi]$ (in this case, a long enough trajectory typically finds the target).

(i) In the $X Y$ model, durations of excursions (as defined in the main text) are broadly distributed. From (A38) it is found that

$$
T_{\text {typ }} \sim \frac{R^{1+\left(d_{f}-1\right) / \beta}}{a^{\left(d_{f}-1\right) / \beta}}
$$

and

$$
G(\eta ; a, r, R)=h(\eta)\left[1-C\left(\frac{a}{r}\right)^{d_{f}-1}\right] .
$$

(ii) In the uniform model, durations of excursions (as defined in the main text) have a finite first moment. Thus,

$$
T_{\text {typ }} \sim \frac{R^{d_{f}}}{a^{d_{f}-1}}
$$

and

$$
G(\eta ; a, r, R)=h(\eta)\left[1-C\left(\frac{a}{r}\right)^{d_{f}-1}\right] .
$$

Concerning the simulation details, at each step we generate the size $l$ of the next jump from the distribution $p(l)$. It is defined by its Fourier transform $\tilde{p}(k)=\exp \left(-k^{\beta}\right)$. The time is incremented by one at each jump for flights and by $l$ for walks.

In two dimensions, in the $X Y$ convention, we first choose randomly a direction ( $x$ or $y$ with a probability $1 / 2$ ) and then perform a jump. In the angular convention, we first randomly choose a direction, that is, an angle in $[0, \pi]$, and then perform a jump along this direction. In the 1D simulation, we take $r=1$ and the domain size $V=R$. In the 2D case, the radius of the target is set to $a=1$, the initial position $r=10$, and targets are distributed on a periodic lattice of mesh size $R$ so that $V=R^{d_{f}}$. In Fig. 2(b), we use $\left(V_{0} ; T_{0}\right)=(100 ; 14180)$ in the case of $X Y$ two-dimensional Lévy walks and $\left(V_{0} ; T_{0}\right)=(200 ; 19540)$ in the case of $X Y$ two-dimensional Lévy flights.

\section{Scaled processes}

In this section, we start with a scale-invariant stochastic process $X^{(0)}(t)$ on a finite domain of fractal dimension $d_{f}$, with vanishing aging exponent $\alpha=0$ and with stationary increments, so

$$
\left\langle\left[X^{(0)}(t+\tau)-X^{(0)}(t)\right]^{2}\right\rangle \propto \tau^{2 / d_{w}^{(0)}},
$$

and we introduce the so-called scaled process $X(t)$, defined by

$$
X(t) \equiv X^{(0)}\left(t^{\beta}\right),
$$

with $\beta>0$. For example, the scaled Brownian motion, which corresponds to the particular case where $X(t)$ is the Brownian motion (and thus with $d_{w}^{(0)}=2$ ), has been used to model anomalous diffusion of passive tracers in complex and biological systems [51]. In what follows, we determine explicitly the typical time $T_{\mathrm{typ}}$ and the distribution $G$ of this scaled process in terms of $d_{f}$ and $d_{w}^{(0)}$ in two ways: (i) by applying directly our 
results on the FPT distribution to the scaled process $X(t)$ and (ii) by deducing them from our results on the FPT distribution of the starting process $X^{(0)}$.

Let us first apply our results on the FPT distribution to the scaled process $X(t)$. The increments of the scaled process can be written as

$$
\begin{aligned}
\left\langle[X(t+\tau)-X(t)]^{2}\right\rangle & =\left\langle\left[X^{(0)}\left((t+\tau)^{\beta}\right)-X^{(0)}\left(t^{\beta}\right)\right]^{2}\right\rangle \\
& \underset{t \rightarrow \infty}{\sim}\left[(t+\tau)^{\beta}-t^{\beta}\right]^{2 / d_{w}^{(0)}} \\
& \underset{t \rightarrow \infty}{\sim} t^{2(\beta-1) / d_{w}^{(0)}} \tau^{2 / d_{w}^{(0)}} .
\end{aligned}
$$

Thus, for the scaled process, the aging exponent $\alpha$ and the walk dimension $d_{w}$ are given by

$$
\alpha=\frac{2(\beta-1)}{d_{w}^{(0)}}, \quad d_{w}=\frac{d_{w}^{(0)}}{\beta},
$$

where we have used the definition

$$
\left\langle[X(t+\tau)-X(t)]^{2}\right\rangle \propto t^{\alpha} \tau^{2 / d_{w}-\alpha}
$$

given in the main text. The transience exponent takes the value

$$
\psi=d_{f}-d_{w}^{(0)}=\psi^{(0)}
$$

and we thus see that the scaled process is noncompact if and only if the original process is also noncompact.

In addition, knowing that the survival probability in infinite space (defined as the probability that the target has not been reached at time $t$ ) can be written as $S_{\infty}(t)=S_{\infty}^{(0)}\left(t^{\beta}\right)$, we have

$$
\theta=\beta \theta^{(0)}=1-\frac{d_{f}}{d_{w}^{(0)}},
$$

where the last equality follows from Appendix A 5. Finally, for the scaled process

$$
T_{\mathrm{typ}}= \begin{cases}R^{d_{w}^{(0)} / \beta} & \text { for } d_{w}^{(0)}>d_{f}(\text { compact case }) \\ \left(\frac{R^{d_{f}}}{a^{d_{f}-d_{w}^{(0)}}}\right)^{1 / \beta} & \text { for } d_{w}^{(0)}<d_{f}(\text { noncompact case })\end{cases}
$$

and the distribution of the rescaled FPT variable $\eta=T / T_{\text {typ }}$ reads [see Eq. (2)]

$$
G(\eta ; a, r, R)= \begin{cases}h(\eta)\left(\frac{r}{R}\right)^{d_{w}^{(0)}-d_{f}} & \text { for } d_{w}^{(0)}>d_{f} \\ h(\eta)\left[1-C\left(\frac{a}{r}\right)^{d_{f}-d_{w}^{(0)}}\right] & \text { for } d_{w}^{(0)}<d_{f},\end{cases}
$$

where we have used the values of the exponents given by Eqs. (B43), (B45), and (B46).

It is instructive to recover this result by starting from the results on the FPT distribution of the original (nonscaled) process $X^{(0)}$. According to Eq. (1), if we define

$$
T_{\mathrm{typ}}^{(0)}= \begin{cases}R^{d_{w}^{(0)}} & \text { for } d_{w}^{(0)}>d_{f} \\ \left(\frac{R^{d_{f}}}{a^{d_{f}-d_{w}^{(0)}}}\right) & \text { for } d_{w}^{(0)}<d_{f},\end{cases}
$$

the distribution of the rescaled FPT $\eta^{(0)}=T / T_{\text {typ }}^{(0)}$ is asymptotically given by

$$
\begin{aligned}
& G^{(0)}\left(\eta^{(0)} ; a, r, R\right) \\
& = \begin{cases}h^{(0)}\left(\eta^{(0)}\right)\left(\frac{r}{R}\right)^{d_{w}^{(0)}-d_{f}} & \text { for } d_{w}^{(0)}>d_{f} \\
h^{(0)}\left(\eta^{(0)}\right)\left[1-C\left(\frac{a}{r}\right)^{d_{f}-d_{w}^{(0)}}\right] & \text { for } d_{w}^{(0)}<d_{f} .\end{cases}
\end{aligned}
$$

The FPT distribution $F(t)$ of the scaled process can be deduced from $F^{(0)}$ as follows:

$$
\begin{aligned}
F(t) & =-\frac{d}{d t} S(t) \\
& =-\frac{d}{d t} S^{(0)}\left(t^{\beta}\right) \\
& =\beta t^{\beta-1} F^{(0)}\left(t^{\beta}\right) .
\end{aligned}
$$

Therefore, the FPT distribution $G(\eta)$ of the rescaled variable $\eta \equiv t / R^{d_{w}}$ corresponding to the scaled process is given by

$$
\begin{aligned}
G(\eta)= & T_{\mathrm{typ}} F(t) \\
= & T_{\mathrm{typ}} \beta t^{\beta-1} F^{(0)}\left(t^{\beta}\right) \\
\sim & \frac{T_{\mathrm{typ}}}{T_{\mathrm{typ}}^{(0)}} t^{\beta-1} h^{(0)}\left(\frac{t^{\beta}}{\left(T_{\mathrm{typ}}^{(0)}\right)^{\beta}}\right) \\
& \times \begin{cases}\left(\frac{r}{R}\right)^{d_{w}^{(0)}-d_{f}} & \text { for } d_{w}^{(0)}>d_{f} \\
{\left[1-C\left(\frac{a}{r}\right)^{d_{f}-d_{w}^{(0)}}\right.} & \text { for } d_{w}^{(0)}<d_{f} .\end{cases}
\end{aligned}
$$

Noting that $T_{\mathrm{typ}}^{\beta}=T_{\mathrm{typ}}^{(0)}$, the result (B48) is recovered.

\section{Case of CTRWs}

In this section, we consider a CTRW on a finite domain of fractal dimension $d_{f}$ : The random walker moves on a network of fractal dimension $d_{f}$, at each time step a neighboring site is chosen at random, and the waiting time $t$ at a given site is drawn from a given distribution $\rho(t)$. For the sake of simplicity, we limit ourselves to the important case of a broad distribution of waiting times

$$
\rho(t) \underset{t \rightarrow \infty}{\sim} \frac{1}{t^{\beta+1}},
$$

with $\beta \in] 0,1[$. Note that in this case, the mean waiting time at each site is infinite, which also implies that the moments of the FPT to a target are infinite. With the prescription given in Appendix A 3, the aging exponent of such a walk is $\alpha=0$.

We introduce the corresponding discrete-time process $X_{n}$, representing the walker position after the $n$th step. Here $X_{n}$ can be seen as a random walk where the walker jumps at all units of time (instead of jumping after a random waiting time). We denote by $d_{w}^{(0)}$ its walk dimension

$$
\left\langle X_{n}^{2}\right\rangle \sim n^{2 / d_{w}^{(0)}} .
$$

Knowing that the number $N(t)$ of jumps of the walker after an observation time $t$ scales as [52]

$$
N(t) \sim t^{\beta},
$$


the walk dimension $d_{w}$ of $X(t)$ can be written as

$$
d_{w}=\frac{d_{w}^{(0)}}{\beta} .
$$

The process $X_{n}$ has by definition stationary increments and we thus have, from Appendix A 5,

$$
\theta^{(0)}=1-\frac{d_{f}}{d_{w}^{(0)}} .
$$

Finally, the persistence exponent $\theta$ is known [15] to be related to $\theta^{(0)}$ by

$$
\theta=\beta \theta^{(0)},
$$

as is found by using the definitions of the persistence exponents $\theta$ and $\theta^{(0)}$ and again the scaling of $N(t)$ given by Eq. (B55). With these results, we finally obtain in the compact case that

$$
T_{\mathrm{typ}}=R^{d_{w}^{(0)} / \beta}
$$

and

$$
G(\eta ; a, r, R)=h(\eta)\left(\frac{r}{R}\right)^{d_{w}^{(0)} \theta^{(0)}}=h(\eta)\left(\frac{r}{R}\right)^{d_{w}^{(0)}-d_{f}} .
$$

Note that the scalings on the geometrical parameters $r$ and $R$ of [21,53] are recovered as specific cases of our general results.

In the noncompact case, the distribution of $\tau_{n}$ has a powerlaw tail

$$
P\left(\tau_{n}\right) \sim \frac{\tilde{\tau}^{\beta}}{\tau_{n}^{1+\beta}} .
$$

We can directly apply Eq. (A38) with $\gamma=\beta$ and $\alpha=0$ :

$$
T_{\text {typ }} \sim R^{d_{w}}\left(\frac{R}{a}\right)^{\psi / \beta} .
$$

Here, because $\psi$ is a geometrical quantity, its value is the same as that of the discrete walk $X_{n}$ :

$$
\psi=d_{f}-d_{w}^{(0)}=d_{f}-d_{w} \beta .
$$

Note that the formula (A48) does not directly apply, because of divergences of both the numerator and denominator of (A45). Finally,

$$
T_{\mathrm{typ}} \sim \frac{R^{d_{f} / \beta}}{a^{d_{f} / \beta-d_{w}}}
$$

and the distribution of the rescaled FPT $\eta=T / T_{\text {typ }}$ reads

$$
G(\eta ; a, r, R)=h(\eta)\left[1-C\left(\frac{a}{r}\right)^{d_{f}-\beta d_{w}}\right] ;
$$

we thus recover the results given in Refs. [21,53] as specific cases of our general results.

\section{Heavy-tailed random acceleration process}

The preceding section explained why usual CTRWs do not exhibit aging for the quantities we focus on; we now turn to an example that combines heavy-tailed effects and aging features. We consider a $d_{f}$-dimensional random acceleration process in discrete time $\mathbf{x}\left(t_{i}\right)$, naturally defined by

$$
\begin{aligned}
& \mathbf{x}\left(t_{i+1}\right)=\mathbf{x}\left(t_{i}\right)+\mathbf{v}\left(t_{i}\right), \\
& \mathbf{v}\left(t_{i+1}\right)=\mathbf{v}\left(t_{i}\right)+\boldsymbol{\xi}\left(t_{i}\right),
\end{aligned}
$$

where $\xi$ is a $d_{f}$-dimensional vector whose coordinates are independent random variables of zero mean and unit variance. Here we assume that the duration of each step $\tau_{i} \equiv t_{i+1}-t_{i}$ is drawn from a Lévy law of the heavy-tailed parameter $\gamma$, i.e., $\rho\left(\tau_{i}\right) \sim_{\tau_{i} \rightarrow \infty} 1 / \tau_{i}^{1+\gamma}$. This obtained process is called the heavy-tailed random acceleration process and combines aging effects and heavy tails by construction.

Since the walk dimension of the standard RAP is $2 / 3$, the walk dimension of the heavy-tailed RAP is $d_{w}=2 / 3 \gamma$. Using the fact that the aging exponent is equal to one for the standard RAP, we obtain an aging exponent for the heavy-tailed RAP $\alpha=\gamma$. Moreover, the transience exponent $\psi$ is not affected by the waiting times, so we still have $\psi=d_{f}-1$. By applying the results of Eq. (A38), we obtain, for $d_{f}>1$,

$$
T_{\text {typ }}=R^{2 / 3 \gamma}\left(\frac{R}{a}\right)^{2\left(d_{f}-1\right) / 3 \gamma} .
$$

Simulations [Fig. 4(h)]. The relation (B68) is checked by means of 2D numerical simulations with $\gamma=0.7$ that are carried out by using directly the discretized Langevin equation (B67). At each time step, we add a normal variable to the velocity, we draw a waiting time $\tau$ from a $\gamma$-stable distribution, and the position of the walker is actualized. Simulations are done in two dimensions, in a confining volume $V=R^{2}$ with periodic boundary conditions. The initial distance between the walker and the target is $r=10$ and the radius of the target is $a=1$.
[1] S. Redner, A Guide to First-Passage Processes (Cambridge University Press, Cambridge, 2001).

[2] R. Metzler, G. Oshanin, and S. Redner, First-Passage Phenomena and Their Applications (World Scientific, Singapore, 2014).

[3] O. Bénichou and R. Voituriez, From first-passage times of random walks in confinement to geometry-controlled kinetics, Phys. Rep. 539, 225 (2014).

[4] S. Rice, Diffusion-Limited Reactions (Elsevier, Amsterdam, 1985).
[5] G. M. Viswanathan, S. V. Buldyrev, S. Havlin, M. G. E. da Luz, E. P. Raposo, and H. E. Stanley, Optimizing the success of random searches, Nature (London) 401, 911 (1999).

[6] A. M. Edwards, R. A. Phillips, N. W. Watkins, M. P. Freeman, E. J. Murphy, V. Afanasyev, S. V. Buldyrev, M. G. E. da Luz, E. P. Raposo, H. E. Stanley, and G. M. Viswanathan, Revisiting Lévy flight search patterns of wandering albatrosses, bumblebees and deer, Nature (London) 449, 1044 (2007). 
[7] M. F. Shlesinger, Mathematical physics: Search research, Nature (London) 443, 281 (2006).

[8] S. Condamin, O. Bénichou, V. Tejedor, R. Voituriez, and J. Klafter, First-passage times in complex scale-invariant media, Nature (London) 450, 77 (2007).

[9] T. Guérin, N. Levernier, O. Bénichou, and R. Voituriez, Mean first-passage times of non-Markovian random walkers in confinement, Nature (London) 534, 356 (2016).

[10] P. L. Krapivsky and S. Redner, Kinetics of a diffusive capture process: Lamb besieged by a pride of lions, J. Phys. A: Math. Gen. 29, 5347 (1996).

[11] S. N. Majumdar and C. Sire, Survival Probability of a Gaussian Non-Markovian Process: Application to the $t=0$ Dynamics of the Ising Model, Phys. Rev. Lett. 77, 1420 (1996).

[12] J. Krug, H. Kallabis, S. N. Majumdar, S. J. Cornell, A. J. Bray, and C. Sire, Persistence exponents for fluctuating interfaces, Phys. Rev. E 56, 2702 (1997).

[13] S. N. Majumdar, Persistence in nonequilibrium systems, Curr. Sci. 77, 370 (1999).

[14] G. Molchan, Maximum of a fractional Brownian motion: Probabilities of small values, Commun. Math. Phys. 205, 97 (1999).

[15] A. J. Bray, S. N. Majumdar, and G. Schehr, Persistence and first-passage properties in nonequilibrium systems, Adv. Phys. 62, 225 (2013).

[16] E. W. Montroll, Random walks on lattices. III. Calculation of first-passage times with application to exciton trapping on photosynthetic units, J. Math. Phys. 10, 753 (1969).

[17] O. Bénichou, C. Chevalier, J. Klafter, B. Meyer, and R. Voituriez, Geometry-controlled kinetics, Nat. Chem. 2, 472 (2010).

[18] T. Guérin, O. Bénichou, and R. Voituriez, Non-Markovian polymer reaction kinetics, Nat. Chem. 4, 568 (2012).

[19] J. H. P. Schulz, E. Barkai, and R. Metzler, Aging Renewal Theory and Application to Random Walks, Phys. Rev. X 4, 011028 (2014).

[20] H. Krüsemann, A. Godec, and R. Metzler, First-passage statistics for aging diffusion in systems with annealed and quenched disorder, Phys. Rev. E 89, 040101 (2014).

[21] S. Condamin, O. Benichou, and J. Klafter, First-Passage Time Distributions for Subdiffusion in Confined Geometry, Phys. Rev. Lett. 98, 250602 (2007).

[22] S. Condamin, V. Tejedor, R. Voituriez, O. Benichou, and J. Klafter, Probing microscopic origins of confined subdiffusion by first-passage observables, Proc. Natl. Acad. Sci. USA 105, 5675 (2008).

[23] L. Berthier and G. Biroli, Theoretical perspective on the glass transition and amorphous materials, Rev. Mod. Phys. 83, 587 (2011).

[24] A. Dechant and E. Lutz, Anomalous Spatial Diffusion and Multifractality in Optical Lattices, Phys. Rev. Lett. 108, 230601 (2012).

[25] A. V. Weigel, B. Simon, M. M. Tamkun, and D. Krapf, Ergodic and nonergodic processes coexist in the plasma membrane as observed by single-molecule tracking, Proc. Natl. Acad. Sci. USA 108, 6438 (2011).

[26] S. M. A. Tabei, S. Burov, H. Y. Kim, A. Kuznetsov, T. Huynh, J. Jureller, L. H. Philipson, A. R. Dinner, and N. F. Scherer, Intracellular transport of insulin granules is a subor- dinated random walk, Proc. Natl. Acad. Sci. USA 110, 4911 (2013).

[27] L. Cipelletti, L. Ramos, S. Manley, E. Pitard, D. A. Weitz, E. E. Pashkovski, and M. Johansson, Universal non-diffusive slow dynamics in aging soft matter, Faraday Discuss. 123, 237 (2003).

[28] J.-P. Bouchaud, Anomalous Relaxation in Complex Systems: From Stretched to Compressed Exponentials (Wiley-VCH, Weinheim, 2008), pp. 327-345.

[29] E. E. Ferrero, K. Martens, and J.-L. Barrat, Relaxation in Yield Stress Systems Through Elastically Interacting Activated Events, Phys. Rev. Lett. 113, 248301 (2014).

[30] H. Krüsemann, A. Godec, and R. Metzler, Ageing first passage time density in continuous time random walks and quenched energy landscapes, J. Phys. A: Math. Theor. 48, 285001 (2015).

[31] S. N. Majumdar, A. Rosso, and A. Zoia, Hitting Probability for Anomalous Diffusion Processes, Phys. Rev. Lett. 104, 020602 (2010).

[32] J. Masoliver, K. Lindenberg, and B. J. West, First-passage times for non-Markovian processes: Correlated impacts on bound processes, Phys. Rev. A, 34, 2351 (1986).

[33] S. V. Buldyrev, M. Gitterman, S. Havlin, A. Y. Kazakov, M. G. E. da Luz, E. P. Raposo, H. E. Stanley, and G. M. Viswanathan, Properties of Lévy flights on an interval with absorbing boundaries, Physica A 302, 148 (2001).

[34] T. W. Burkhardt, The random acceleration process in bounded geometries, J. Stat. Mech. (2007) P07004.

[35] R. Galloti, A. Bazzani, S. Rambaldi, and M. Barthelemy, A stochastic model of randomly accelerated walkers for human mobility, Nat. Commun. 7, 12600 (2016).

[36] A. V. Chechkin, R. Metzler, V. Y. Gonchar, J. Klafter, and L. V. Tanatarov, First passage and arrival time densities for Lévy flights and the failure of the method of images, J. Phys. A: Math. Gen. 36, L537 (2003).

[37] Y. Meroz, I. M. Sokolov, and J. Klafter, Distribution of firstpassage times to specific targets on compactly explored fractal structures, Phys. Rev. E 83, 020104 (2011).

[38] D. Ben-Avraham and S. Havlin, Diffusion and Reactions in Fractals and Disordered Systems (Cambridge University Press, Cambridge, 2000).

[39] A. T. A. Wood and G. Chan, Simulation of stationary Gaussian processes in $[0,1]^{d}$, J. Comput. Graph. Stat. 3, 409 (1994).

[40] R. B. Davies and D. Harte, Tests for Hurst effect, Biometrika 74, 95 (1987).

[41] C. Dietrich and G. N. Newsam, Fast and exact simulation of stationary Gaussian processes through circulant embedding of the covariance matrix, SIAM J. Sci. Comput. 18, 1088 (1997).

[42] J. Masoliver and J. M. Porrà, Exact solution to the exit-time problem for an undamped free particle driven by Gaussian white noise, Phys. Rev. E 53, 2243 (1996).

[43] D. J. Bicout and T. W. Burkhardt, Absorption of a randomly accelerated particle: Gambler's ruin in a different game, J. Phys. A: Math. Gen. 33, 6835 (2000).

[44] G. M. Viswanathan, M. G. Da Luz, E. P. Raposo, and H. E. Stanley, The Physics of Foraging: An Introduction to Random Searches and Biological Encounters (Cambridge University Press, Cambridge, 2011). 
[45] V. Tejedor, O. Bénichou, R. Metzler, and R. Voituriez, Residual mean first-passage time for jump processes: Theory and applications to Lévy flights and fractional Brownian motion, J. Phys. A: Math. Theor. 44, 255003 (2011).

[46] S. Redner, A Guide to First-Passage Processes (Cambridge University Press, Cambridge, 2001).

[47] S. V. Buldyrev, S. Havlin, A. Y. Kazakov, M. G. E. da Luz, E. P. Raposo, H. E. Stanley, and G. M. Viswanathan, Average time spent by Lévy flights and walks on an interval with absorbing boundaries, Phys. Rev. E 64, 041108 (2001).

[48] V. Zaburdaev, S. Denisov, and J. Klafter, Lévy walks, Rev. Mod. Phys. 87, 483 (2015).
[49] N. Korabel and E. Barkai, Anomalous infiltration, J. Stat. Mech. (2011) P05022.

[50] V. Zaburdaev, I. Fouxon, S. Denisov, and E. Barkai, Superdiffusive Dispersals Impart the Geometry of Underlying Random Walks, Phys. Rev. Lett. 117, 270601 (2016).

[51] H. Safdari, A. V. Chechkin, G. R. Jafari, and R. Metzler, Aging scaled Brownian motion, Phys. Rev. E 91, 042107 (2015).

[52] J.-P. Bouchaud and A. Georges, Anomalous diffusion in disordered media: Statistical mechanisms, models and physical applications, Phys. Rep. 195, 127 (1990).

[53] B. Meyer, C. Chevalier, R. Voituriez, and O. Bénichou, Universality classes of first-passage-time distribution in confined media, Phys. Rev. E 83, 051116 (2011). 